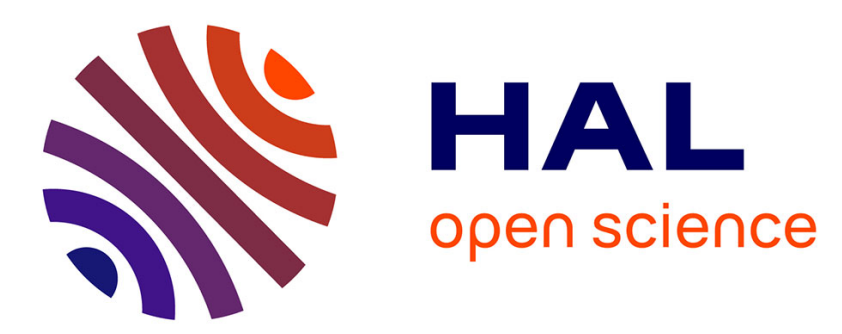

\title{
Third-order Volterra MVDR beamforming for non-Gaussian and potentially non-circular interference cancellation
}

Pascal Chevalier, Jean-Pierre Delmas, Mustapha Sadok

\section{- To cite this version:}

Pascal Chevalier, Jean-Pierre Delmas, Mustapha Sadok. Third-order Volterra MVDR beamforming for non-Gaussian and potentially non-circular interference cancellation. IEEE Transactions on Signal Processing, 2018, 66 (18), pp.4766-4781. 10.1109/TSP.2018.2860551 . hal-01869124

\section{HAL Id: hal-01869124 \\ https://hal.science/hal-01869124}

Submitted on 6 Sep 2018

HAL is a multi-disciplinary open access archive for the deposit and dissemination of scientific research documents, whether they are published or not. The documents may come from teaching and research institutions in France or abroad, or from public or private research centers.
L'archive ouverte pluridisciplinaire HAL, est destinée au dépôt et à la diffusion de documents scientifiques de niveau recherche, publiés ou non, émanant des établissements d'enseignement et de recherche français ou étrangers, des laboratoires publics ou privés. 


\title{
Third-order Volterra MVDR beamforming for non-Gaussian and potentially non-circular interference cancellation
}

\author{
Pascal Chevalier, Jean-Pierre Delmas, and Mustapha Sadok
}

\begin{abstract}
Linear beamformers are optimal, in a mean square (MS) sense, when the signal of interest (SOI) and observations are jointly Gaussian and circular. Otherwise, linear beamformers become sub-optimal. When the SOI and observations are zero-mean, jointly Gaussian and non-circular, optimal beamformers become widely linear (WL). They become non-linear with a structure depending on the unknown joint probability distribution of the SOI and observations when the latter are jointly non-Gaussian, assumption which is very common in radiocommunications. In this context, the paper aims at introducing, for small-scale systems, third-order Volterra minimum variance distortionless response (MVDR) beamformers, for the reception of a SOI, whose waveform is unknown but whose steering vector is known, corrupted by non-Gaussian and potentially non-circular interference, omnipresent in practical situations. Properties, performance, complexity and adaptive implementation of these beamformers in the presence of non-Gaussian and potentially non-circular interference are analyzed in this paper. These new beamformers are shown to always improve, in the steady state, the performance of Capon beamformer for non-gaussian/circular interference, whereas some of them improve the performance of the WL MVDR beamformer for non-Gaussian/non-circular interference. These new beamformers open new perspectives for spectrum monitoring of non-Gaussian signals and for radiocommunication networks using such signals.
\end{abstract}

Index Terms-Non-linear, non-Gaussian, non-circular, widely linear, third-order Volterra, interference, MVDR, beamforming, spectrum monitoring, passive listening.

\section{INTRODUCTION}

B EAMFORMING plays an important role in many applications such as radar, sonar, satellite communications, radiocommunications, acoustic or spectrum monitoring [1]. It allows to optimize, by a linear filtering of the observations, the reception of a SOI potentially corrupted by interference. It consists to steer a beam in the SOI direction while forming spatial holes in the interference directions. The most popular receive beamformer has been introduced by Capon and al. [2] at the end of the sixties and corresponds to the MVDR beamformer. It consists to minimize the output power under a linear constraint of non-distortion of the SOI. Its implementation only requires the a priori knowledge or estimation of the steering vector of the SOI, hence its great interest for spectrum monitoring or passive listening in particular. It corresponds to a particular case of linearly constrained minimum variance (LCMV) beamformer [3] whose equivalent unconstrained form is the generalized sidelobe canceller (GSC) introduced in [4]. As the Capon beamformer is not robust to errors in the SOI steering vector when it is adapted in the presence of the SOI, most of the papers about beamforming written these last decades, have concerned the development of numerous beamforming concepts robust to errors in the SOI steering vector [5]. However, the available robust beamformers are still linear beamformers but with additional constraints allowing to preserve the SOI contribution in the presence of errors on the SOI steering vector.

Nevertheless it is now well-known [6] that the optimal beamformer, in a mean square (MS) sense, whose output corresponds to the conditional expectation of the SOI with respect to the observations, is linear only when the SOI and the observations are jointly Gaussian and circular [7]. Linear beamformers then become sub-optimal for non-Gaussian and/or non-circular SOI and/or observations, omnipresent in radiocommunications in particular. Indeed, most of digital communications signals are non-Gaussian and many of them are non-circular either at the second order (SO) and/or at a higher order (HO). For example, an amplitude shift keying (ASK) signal is non-Gaussian and at least noncircular at all even orders. A phase shift keying signal with $M$ states ( $M$-PSK) is non-Gaussian and non-circular at an order $2 q$ such that $2 q \geq M$ [8]. A square quadrature amplitude modulated (QAM) signal with $4 M^{2}$ state $\left(4 M^{2}\right.$ QAM) is non-Gaussian and at least fourth-order (FO) noncircular. In this context, non linear beamformers become of great interest for both radiocommunications and spectrum monitoring of radiocommunications.

More precisely, when the SOI and observations are zeromean, jointly Gaussian but non-circular, the optimal beamformer becomes WL [6], which corresponds to a particular non-linear structure weighting linearly and independently the observations and their complex conjugate. For this reason, a WL MVDR beamformer (called WL MVDR ${ }_{1}$ ), exploiting the potential SO non-circularity of the interference only, has been introduced recently in [9] for spectrum monitoring of radiocommunications and its implementation has been discussed in [10]. To take into account the potential SO non-circularity of both the SOI and the interference, 
a second WL MVDR beamformer (called WL MVDR 2 ) has been further introduced in [11], [12]. This beamformer allows us to implement the WL minimum mean square error (MMSE) beamformer [13], without requiring the a priori knowledge of the SOI, but only from the a priori knowledge or estimation of both the steering vector and the SO noncircularity coefficient of the SOI. Note that for a strong SO circular SOI, the WL $\mathrm{MVDR}_{2}$ beamformer coincides with the WL MVDR 1 beamformer [12]. A limitation of the WL $\mathrm{MVDR}_{2}$ beamformer is that it is not robust to errors in either the SOI steering vector or the SOI SO non-circularity coefficient, when it is adapted in the presence of the SOI. For this reason, since a decade, most of the papers about WL beamforming have concerned the robustness increase of the WL MVDR 2 beamformer, through the development of numerous robust WL beamforming concepts [14]-[18]. The numerous available robust WL beamformers are still WL beamformers but with additional constraints allowing to preserve the SOI contribution in the presence of errors on the steering vector and/or the SO non-circularity coefficient of the SOI. Note that alternative WL beamformers have also been proposed recently to mitigate RF impairments [19], for reduced-rank beamforming [20] in massive MIMO systems and also for binaural noise reduction in acoustic [21], [22].

Moreover, when the SOI and observations are jointly nonGaussian (jointly circular or not), the optimal beamformer becomes a non-linear function of the observations, which depends on the joint probability distribution of the SOI and the observed data. However in practice, this probability distribution is generally not known a priori. A first philosophy then consists in trying to estimate it in order to optimize the non-linearity of the beamformer. This estimation may be implemented through stochastic techniques, based, for example, on particle filtering [23], [24] or through a parametric model of the non-Gaussian observations, such as the Gaussian mixture model [25], well-suited to model non-Gaussian/non-circular noise [26]. However, in all cases, this philosophy is generally costly and difficult to implement. A second philosophy, much easier to implement, consists in imposing a particular non-linear structure to the beamformer, including the linear one, and to compute a beamformer (MVDR for example) having this imposed structure. Although sub-optimal, the considered non-linear beamformer is built to generate a performance improvement with respect to the linear one in non-Gaussian contexts. Such an approach, based on the constant modulus criterion, has been investigated in [27] for non-Gaussian noise rejection in the context of satellite communications by using the concept of radial basis functions (RBF) beamforming. However, although powerful for non-Gaussian noise rejection, the RBF beamformer may have limited performance for Gaussian and circular noise since it does not include the linear structure.

A particular non-linear structure, including both the linear and the WL structures, corresponds to the pth-order $(p \geq 2)$ complex Volterra structure [28], [29]. Such a structure is able to improve the performance of linear beamformers in non-Gaussian and potentially non-circular contexts, by exploiting both the non-Gaussiannity and the complete potential non-circularity of the observations up to the order $2 p$. Let us recall that Volterra filtering [30] has been considered in signal processing for a long time for many applications such as for example detection and estimation [31], system identification [32], echo cancellation [33] or non linear channel equalization [34] but mainly for real-valued observations. The main use of Volterra filtering for complex data concerns both the modeling and the predistortion processing of the baseband input-output relationship of power amplifiers operating close to saturation for power efficiency in radiocommunications [35], [36]. The scarce other works about complex Volterra filtering mainly concern blind identification of some linear-quadratic systems [37], MS estimation and detection from linearquadratic [38] or pth-order systems [28], [29], beamforming [39], [40] and single antenna interference cancellation (SAIC) [41]. [39] introduces a particular third-order Volterra MVDR beamformer for non-Gaussian interference rejection improvement. However, this beamformer does not include the WL structure, does not take into account the potential non-circularity of the interference and may generate lower performance than the WL beamfomers. In contrast, [40] and [41] introduce more general third-order Volterra beamformers, exploiting both the non-Gaussiannity and the potential non-circularity of the interference. However [40] concerns coded division multiple access (CDMA) cellular networks, whereas [41] assumes only ASK signals and one reception antenna. Both systems use a MMSE approach, optimized for demodulation purpose and their implementation requires a training sequence. They cannot be used for spectrum monitoring of general radiocommunication signals.

In this context, the first purpose of this paper is to introduce several third-order Volterra MVDR beamformers for the reception of a SOI, whose waveform is unknown but whose steering vector is known, corrupted by potentially non-Gaussian and non-circular interference. All these beamformers are third-order extensions of the Capon beamformer [2], whereas some of them are third-order extensions of the WL MVDR 1 beamformer [9] or are alternatives to the third-order Volterra beamformer introduced in [39]. All the proposed beamformers exploit the potential non-Gaussian character of the interference, whereas some of them exploit, in addition, their non-circularity up to order 4 or 6 . It is important to note that the proposed beamformers have no interest for large-scale systems, such as massive MIMO systems for $5 \mathrm{G}$ mobile cellular networks, for which the linear beamformers are quasi-optimal since the sources can be assumed to be approximately orthogonal to each other for the array. On the contrary, the proposed beamformers are mainly developed for small-scale systems, with a small 
number of antennas and low spatial aperture in number of wavelength, which are low spatial resolution systems for which the Capon beamformer has limited performance in the presence of interference. For such systems the idea is to replace the missing hardware (or antennas) by clever software with a moderate complexity, as shown in the paper, to improve the interference cancellation. Note that most of the array processing systems working in the HF (3 - $30 \mathrm{MHz}$ ), VHF (30 - $300 \mathrm{MHz}$ ) or UHF band (300 $\mathrm{MHz}-3 \mathrm{Ghz}$ ), for which the large size of the wavelength $(0.10 \mathrm{~m} \leq \lambda \leq 100 \mathrm{~m})$, jointly with the limited size of the carrier system (vehicle, plane, manpack, infrastructure..), limit the number of antennas, are small-scale systems. This is currently the case and this will remain the case in the future, since the allocated wavelength of these systems should not change. This concerns numerous applications such as $2 \mathrm{G} / 3 \mathrm{G} / 4 \mathrm{G}$ cellular mobile communications and metrology, airborne communications and spectrum monitoring, military tactical communications, electronic warfare, naval communications... The analysis of the properties, performance, complexity and adaptive implementation of the proposed third-order beamformers are the second purpose of this paper. For interference having a high spatial correlation with the SOI, usual for small-scale systems, it is shown in the paper that the best proposed third-order beamformers may strongly improve the performance of the Capon and WL MVDR 1 beamformer for non-Gaussian/circular and non-Gaussian/non-circular interference respectively, and the Capon and third-order Volterra beamformer [39] performance for non-circular interference.

The proposed beamformers open new perspectives for spectrum monitoring of non-Gaussian and non-circular signals and for radiocommunication networks using such signals in particular. Note that the FO non-circularity of observations has been used by a WL MMSE beamformer in [42] to compensate I/Q imbalance effects at reception but not to improve the steady-state performance of WL beamformers. In addition, the non-Gaussiannity and both the sub-Gaussiannity and non-circularity of observations has already been used in [43] and [44], respectively, through the development of the linear minimum dispersion beamformer (MDB) and the WL MDB respectively, to boost the convergence speed of linear and WL beamformers respectively, but not to improve their steady-state performance. Finally note that some preliminary results of the paper have been presented in [45], but without any complexity and analytical performance analysis and with no comparison with nonlinear beamformers of the literature.

After the introduction of some hypotheses, data statistics and problem formulation are given in Section II. Section III introduces the new third-order Volterra MVDR beamformers, jointly with their equivalent GSC structure and the related generic output signal to interference plus noise ratio (SINR) performance. An analytical performance analysis of some of the proposed MVDR beamformers, in the presence of a single interference, is presented in Section IV. The adaptive implementation of the proposed beamformers is briefly investigated in Section $\mathrm{V}$, whereas some complexity elements of the latter are briefly described in Section VI. Finally Section VII concludes this paper.

The following notations are used throughout the paper. Matrices and vectors are represented by bold upper case and bold lower case characters, respectively. Vectors are by default in column orientation, while $T, H$ and $*$ stand for transpose, conjugate transpose and conjugate, respectively. $\mathrm{E}($.$) is the expectation operator. \mathbf{I}_{p}, \mathbf{0}_{p}$ and $\mathbf{O}_{p, q}$ are the identity matrix of order $p$, the null vector of size $p$ and the null matrix of order $p \times q$, respectively. $\operatorname{Diag}\left(\mathbf{A}_{1}, \ldots, \mathbf{A}_{q}\right)$ represents a block diagonal matrix of diagonal elements $\mathbf{A}_{1}, . ., \mathbf{A}_{q} . \otimes$ denotes the Kronecker product and $\mathbf{A}^{\otimes q}$ means $\mathbf{A} \otimes \mathbf{A} \ldots \otimes \mathbf{A}$ with $q-1$ Kronecker products.

\section{HYPOTHESES, DATA STATISTICS AND PROBLEM FORMULATION}

\section{A. Hypotheses}

We consider an array of $N$ narrowband sensors and we denote by $\mathbf{x}(t)$ the vector of the complex amplitudes of the signals at the output of these sensors. Each sensor is assumed to receive the contribution of an SOI corrupted by interference and a background noise. Under these assumptions, the observation vector $\mathbf{x}(t)$ can be written as follows

$$
\mathbf{x}(t)=s(t) \mathbf{s}+\mathbf{n}(t) \in \mathbb{C}^{N} .
$$

Here, $s(t)$ and $\mathbf{s}$ correspond to the complex envelope, assumed zero-mean, and the steering vector, assumed perfectly known, of the SOI respectively. The vector $\mathbf{n}(t)$ is the total noise vector, containing the background noise and the interference, and assumed to be zero-mean, potentially nonGaussian and non-circular, and independent of $s(t)$.

\section{B. Data statistics}

1) Presentation: Since vector $\mathbf{x}(t)$ is generally nonstationary in radiocommunications or spectrum monitoring contexts, the statistics of $\mathbf{x}(t)$ which are considered in this paper correspond to the temporal mean, in the variable $t$, of the moments of $\mathbf{x}(t)$ up to the 6th-order. To illustrate these moments, which will be used in Subsection IV-B, we compute in this section the $n$ th-order moments $(1 \leq n \leq 6)$ of a scalar signal $u(t)$, which may correspond to the SOI $s(t)$, an interference or a background noise. Furthermore, as in practice the probability distributions of $u(t)$ are often symmetric, for which the odd-order moments are zero, we limit the computation to $n=2 p, 1 \leq p \leq 3$. As $\left.\mathrm{E}\left[u^{2 p-q}(t) u^{* q}(t)\right]=\left(\mathrm{E}\left[u^{q}(t) u^{*(2 p-q)}(t)\right)\right]\right)^{*}$, the $2 p$ thorder moments of $u(t)$ are completely defined from the $p+1$ moments $\mathrm{E}\left[u^{q}(t) u^{*(2 p-q)}(t)\right], p \leq q \leq 2 p$. We will see in Section III that the proposed beamformers exploit the information contained in the temporal mean $<$ $\mathrm{E}\left[u^{q}(t) u^{*(2 p-q)}(t)\right]>$ of the statistics $\mathrm{E}\left[u^{q}(t) u^{*(2 p-q)}(t)\right]$ 
for $0 \leq q \leq 2 p$ and $1 \leq p \leq 3$, where $<$. $>$ is the temporal mean operation, in $t$, over the observation duration. In particular, $\pi_{u} \stackrel{\text { def }}{=}<\mathrm{E}\left|u^{2}(t)\right|>$ is the input power of the signal $u(t)$ seen by the beamformer.

The real-valued FO and sixth-order (SIO) circular coefficient of $u(t)$ are simply denoted by $\kappa_{u, c}$ and $\chi_{u, c}$ respectively, defined by

$$
\kappa_{u, c} \stackrel{\text { def }}{=} \frac{<\mathrm{E}\left|u^{4}(t)\right|>}{\left(<\mathrm{E}\left|u^{2}(t)\right|>\right)^{2}} \text { and } \chi_{u, c} \stackrel{\text { def }}{=} \frac{<\mathrm{E}\left|u^{6}(t)\right|>}{\left(<\mathrm{E}\left|u^{2}(t)\right|>\right)^{3}} .
$$

The generally complex-valued SO, FO and SIO non-circular coefficients of $u(t)$ are simply denoted by $\gamma_{u}, \kappa_{u, n c, i}$ and $\chi_{u, n c, i}$, respectively, defined by

$$
\begin{gathered}
\gamma_{u} \stackrel{\text { def }}{=} \frac{<\mathrm{E}\left[u^{2}(t)\right]>}{<\mathrm{E}\left|u^{2}(t)\right|>}, \\
\kappa_{u, n c, i} \stackrel{\text { def }}{=} \frac{<\mathrm{E}\left[u^{5-i}(t) u^{*(i-1)}(t)\right]>}{\left(<\mathrm{E}\left|u^{2}(t)\right|>\right)^{2}}, i=1,2 \\
\chi_{u, n c, i} \stackrel{\text { def }}{=} \frac{<\mathrm{E}\left[u^{7-i}(t) u^{*(i-1)}(t)\right]>}{\left(<\mathrm{E}\left|u^{2}(t)\right|>\right)^{3}}, i=1,2,3 .
\end{gathered}
$$

It is easy to verify that $\left|\gamma_{u}\right| \leq 1$. The signal $u(t)$ is seen as SO circular by the proposed beamformers if $\gamma_{u}=0$. It is seen as SO non-circular otherwise. When $u(t)$ is real-valued to within a phase term, it is called rectilinear and $\left|\gamma_{u}\right|=1$. In a similar way, it is easy to verify that $\left|\kappa_{u, n c, i}\right| \leq \kappa_{u, c}, i=$ 1,2 and $\left|\chi_{u, n c, i}\right| \leq \chi_{u, c}, i=1,2,3$. The signal $u(t)$ is seen as FO and SIO circular if $\kappa_{u, n c, i}=0, i=1,2$ and $\chi_{u, n c, i}=$ $0, i=1,2,3$, respectively. It is seen as FO and SIO noncircular otherwise respectively. For a rectilinear signal $u(t)$, $\left|\kappa_{u, n c, i}\right|=\kappa_{u, c}, i=1,2$ and $\left|\chi_{u, n c, i}\right|=\chi_{u, c}, i=1,2,3$. Note finally that for any signal $u(t)$, it is easy to verify that $1 \leq \kappa_{u, c}^{2} \leq \chi_{u, c}$ and $\left|\kappa_{u, n c, i}^{2}\right| \leq \chi_{u, c}, i=1,2$.

2) Particular cases: To quantify and illustrate, in Subsection IV-B, the performance of the proposed beamformers, we consider hereafter three particular cases of signal $u(t)$.

In the first case, $u(t)$ is assumed to be zero-mean stationary and Gaussian. It is then straightforward to prove, from SO, FO and SIO cumulants expressions [46], that the FO coefficients are given by

$$
\kappa_{u, c}=2+\left|\gamma_{u}\right|^{2}, \quad \kappa_{u, n c, 1}=3 \gamma_{u}^{2} \text { and } \kappa_{u, n c, 2}=3 \gamma_{u},
$$

whereas the SIO coefficients are given by

$$
\begin{gathered}
\chi_{u, c}=3\left(2+3\left|\gamma_{u}\right|^{2}\right), \\
\chi_{u, n c, 1}=15 \gamma_{u}^{3}, \chi_{u, n c, 2}=15 \gamma_{u}^{2}, \chi_{u, n c, 3}=3 \gamma_{u}\left(4+\left|\gamma_{u}\right|^{2}\right) .
\end{gathered}
$$

Expressions (6), (7) and (8) show that for a zero-mean stationary Gaussian signal, the FO and SIO circular coefficients of $u(t)$ are increasing functions of $\left|\gamma_{u}\right|$ such that $2 \leq \kappa_{u, c} \leq 3$ and $6 \leq \chi_{u, c} \leq 15$. In particular, $\left(\kappa_{u, c}, \chi_{u, c}\right)=(2,6)$ for a circular $\left(\gamma_{u}=0\right)$ Gaussian signal, whereas $\left(\kappa_{u, c}, \chi_{u, c}\right)=(3,15)$ for a rectilinear $\left(\left|\gamma_{u}\right|=1\right)$ Gaussian signal. We deduce from this result that a zero- mean stationary signal such that $0 \leq \kappa_{u, c}<2$ or $3<\kappa_{u, c}$ or $0 \leq \chi_{u, c}<6$ or $15<\chi_{u, c}$ is necessarily non-Gaussian, which means that $\kappa_{u, c}$ and $\chi_{u, c}$ are measures of nonGaussianity. Moreover, expressions (6), (7) and (8) show that for zero-mean stationary Gaussian signals, SO, FO and SIO circularity are equivalent and, as the modulus of SO, FO and SIO non-circular coefficients are all maximal for rectilinear signals, full SO, FO and SIO non-circularity are also equivalent. Finally we verify that $1 \leq \kappa_{u, c}^{2} \leq \chi_{u, c}$ whatever $\gamma_{u}$.

In the second case, $u(t)$ corresponds to the complex envelope of a digital linearly modulated signal, defined by:

$$
u(t)=\mu e^{i \phi} \sum_{n} a_{n} v\left(t-n T-t_{0}\right) .
$$

Here, the $a_{n}$ 's are i.i.d. zero-mean random variables corresponding to the transmitted symbols, $T$ is the symbol duration, $t_{0}$ is the initial sampling time, $v(t)$ is a real-valued pulse shaping filter, $\mu$ is a constant controlling the amplitude of $u(t)$ and $\phi$ is a phase term. It is easy to verify that the SO non-circular coefficient of $u(t)$ is given by

$$
\gamma_{u}=e^{i 2 \phi} \gamma_{a}
$$

where $\gamma_{a}$ is the SO non-circular coefficient of the symbol $a_{n}$. Moreover, in the particular case of a filter $v(t)$ which is rectangular over the symbol duration $T$ and assuming an infinite observation duration, it is straightforward to prove that $\kappa_{u, c}=\kappa_{a, c}, \kappa_{u, n c, 1}=e^{4 i \phi} \kappa_{a, n c, 1}, \kappa_{u, n c, 2}=e^{2 i \phi} \kappa_{a, n c, 2}$, $\chi_{u, c}=\chi_{a, c}, \chi_{u, n c, 1}=e^{6 i \phi} \chi_{a, n c, 1}, \chi_{u, n c, 2}=e^{4 i \phi} \chi_{a, n c, 2}$ and $\chi_{u, n c, 3}=e^{2 i \phi} \chi_{a, n c, 3}$ where $\kappa_{a, c}, \kappa_{a, n c, 1}, \kappa_{a, n c, 2}, \chi_{a, c}$, $\chi_{a, n c, 1}, \chi_{a, n c, 2}$ and $\chi_{a, n c, 3}$ are the FO and SIO coefficients of the symbol $a_{n}$. This results mean that, for a non-filtered linearly modulated signal, the coefficients of $u(t)$ and $e^{i \phi} a_{n}$ coincide both at FO and SIO. To evaluate the non-Gaussian and potentially non-circular nature of some constellations used in practice, Table 1 summarizes the value of the $\mathrm{SO}, \mathrm{FO}$ and SIO coefficients of the symbol $a_{n}$, when the constellation is BPSK, QPSK, 8-PSK, 4-ASK, 16-QAM, circular Gaussian (C-Gaus) and rectilinear Gaussian (RGaus) respectively.

\begin{tabular}{|c|c|c|c|c|c|c|c|}
\hline & BPSK & QPSK & 8-PSK & 4-ASK & 16-QAM & C-Gaus & R-Gaus \\
\hline$\gamma_{a}$ & 1 & 0 & 0 & 1 & 0 & 0 & 1 \\
\hline$\kappa_{a, c}$ & 1 & 1 & 1 & $41 / 25$ & $33 / 25$ & 2 & 3 \\
\hline$\kappa_{a, n c, 1}$ & 1 & 1 & 0 & $41 / 25$ & $-17 / 25$ & 0 & 3 \\
\hline$\kappa_{a, n c, 2}$ & 1 & 0 & 0 & $41 / 25$ & 0 & 0 & 3 \\
\hline$\chi_{a, c}$ & 1 & 1 & 1 & $73 / 25$ & $49 / 25$ & 6 & 15 \\
\hline$\chi_{a, n c, 1}$ & 1 & 0 & 0 & $73 / 25$ & 0 & 0 & 15 \\
\hline$\chi_{a, n c, 2}$ & 1 & 1 & 0 & $73 / 25$ & $-33 / 25$ & 0 & 15 \\
\hline$\chi_{a, n c, 3}$ & 1 & 0 & 0 & $73 / 25$ & 0 & 0 & 15 \\
\hline
\end{tabular}

Table $1 \mathrm{SO}$, FO and SIO coefficients of several constellations

Finally, in the third case, $u(t)=r(t) e^{i \theta(t)}$ corresponds to a zero-mean stationary white signal whose amplitude is impulsive, where $r(t)$ and $\theta(t)$ are statistically independent stationary real-valued processes, $r(t)$ is Bernoulli distributed, taking amplitude $\mu$ with probability $p$ and 0 with probability $1-p$, and $\theta(t)$ is uniformly distributed either on $[0,2 \pi]$, or on the set of two values $\left\{\theta_{0}, \theta_{0}+\pi\right\}$ where 
$\theta_{0}$ is constant. In the first case, $u(t)$ is $n$ th-order circular whatever $n$, whereas in the second case $u(t)$ is rectilinear. In both cases, we obtain :

$$
\kappa_{u, c}=1 / p \text { and } \chi_{u, c}=1 / p^{2},
$$

whereas in the second case, we obtain

$$
\begin{aligned}
\kappa_{u, n c, 1} & =(1 / p) e^{i 4 \theta_{0}}, \quad \kappa_{u, n c, 2}=(1 / p) e^{i 2 \theta_{0}}, \\
\chi_{u, n c, 1} & =\left(1 / p^{2}\right) e^{i 6 \theta_{0}}, \chi_{u, n c, 2}=\left(1 / p^{2}\right) e^{i 4 \theta_{0}}, \\
\chi_{u, n c, 3} & =\left(1 / p^{2}\right) e^{i 2 \theta_{0}} .
\end{aligned}
$$

\section{Problem formulation}

1) Optimal or MMSE beamformer: It is well-known [6] that the optimal estimate, $\widehat{s}_{\mathrm{MMSE}}(t)$, of $s(t)$, in a MS sense, from the observation vector $\mathbf{x}(t)$, is the conditional expectation of $s(t)$ with respect to $\mathbf{x}(t)$, defined by

$$
\widehat{s}_{\mathrm{MMSE}}(t)=\mathrm{E}[s(t) \mid \mathbf{x}(t)] \text {. }
$$

Let us consider an $N \times 1$ spatial filter $\mathbf{w}_{f}$ such that $\mathbf{w}_{f}^{H} \mathbf{s}=1$. Applying the linearity property of the conditional expectation (14) to $s(t)=\mathbf{w}_{f}^{H} \mathbf{x}(t)-\mathbf{w}_{f}^{H} \mathbf{n}(t)$, deduced from the model (1), we obtain:

$$
\begin{aligned}
\widehat{s}_{\mathrm{MMSE}}(t) & =\mathbf{w}_{f}^{H} \mathbf{x}(t)-\mathbf{w}_{f}^{H} \mathrm{E}[\mathbf{n}(t) \mid \mathbf{x}(t)] \\
& =s(t)+\mathbf{w}_{f}^{H}(\mathbf{n}(t)-\mathrm{E}[\mathbf{n}(t) \mid \mathbf{x}(t)]) .
\end{aligned}
$$

Expressions (14) and (15) show that $\widehat{s}_{\mathrm{MMSE}}(t)$, and then the optimal beamformer, depends on the probability distribution of $(s(t), \mathbf{x}(t))$ or $(\mathbf{n}(t), \mathbf{x}(t))$, and optimally exploits the potential non-Gaussian and non-circular characters of both the SOI $s(t)$ and the total noise $\mathbf{n}(t)$. However, these probability distributions are generally unknown in practice and sub-optimal approaches must be considered.

2) MVDR beamformer exploiting the total noise statistics only: The knowledge of $\mathbf{s}$ allows for the projection of $\mathbf{x}(t)$ on $\mathbf{s}$ and its orthogonal space $\operatorname{span}(\mathbf{s})^{\perp}$, giving rise to $\mathbf{x}_{s}(t) \stackrel{\text { def }}{=}\left(\mathbf{s}^{H} \mathbf{s}\right)^{-1} \mathbf{s s}^{H} \mathbf{x}(t) \stackrel{\text { def }}{=} \boldsymbol{\Pi}_{s} \mathbf{x}(t)$ and $\mathbf{x}_{s^{\perp}}(t) \stackrel{\text { def }}{=}$ $\left[\mathbf{I}-\left(\mathbf{s}^{H} \mathbf{s}\right)^{-1} \mathbf{s s}^{H}\right] \mathbf{x}(t) \stackrel{\text { def }}{=} \boldsymbol{\Pi}_{s} \perp \mathbf{x}(t)$, respectively such that:

$$
\mathbf{x}(t)=\mathbf{x}_{s}(t)+\mathbf{x}_{s^{\perp}}(t)=s(t) \mathbf{s}+\mathbf{n}_{s}(t)+\mathbf{n}_{s^{\perp}}(t),
$$

where $\mathbf{n}_{s}(t) \stackrel{\text { def }}{=} \boldsymbol{\Pi}_{s} \mathbf{n}(t)$ and $\mathbf{n}_{s^{\perp}}(t) \stackrel{\text { def }}{=} \boldsymbol{\Pi}_{s^{\perp}} \mathbf{n}(t)=\mathbf{x}_{s^{\perp}}(t)$.

Let us now consider an estimate, $\widehat{s}(t)$, of $s(t)$ from an arbitrary function of $\mathbf{x}(t), f(\mathbf{x}(t))$. If we impose to $f(\mathbf{x}(t))$ to be linear with respect to $s(t)$ and to generate no SOI distorsion, $\widehat{s}(t)=f(\mathbf{x}(t))$ becomes linear with respect to $\mathbf{x}_{s}(t)$ and takes the form:

$$
\begin{aligned}
\widehat{s}(t) & =\mathbf{w}_{f}^{H} \mathbf{x}_{s}(t)-g\left(\mathbf{x}_{s^{\perp}}(t)\right) \\
& =s(t)+\left(\mathbf{s}^{H} \mathbf{s}\right)^{-1} \mathbf{s}^{H} \mathbf{n}(t)-g\left(\mathbf{n}_{s^{\perp}}(t)\right) .
\end{aligned}
$$

The MVDR beamformer having the structure (17) is the one which minimizes the MS error $\mathrm{E}\left[\mid\left(\mathbf{s}^{H} \mathbf{s}\right)^{-1} \mathbf{s}^{H} \mathbf{n}(t)-\right.$ $\left.\left.g\left(\mathbf{n}_{s^{\perp}}(t)\right)\right|^{2}\right]$. The solution to this problem is the conditional expectation: $g_{\mathrm{MVDR}}\left(\mathbf{n}_{s^{\perp}}(t)\right)=\mathrm{E}\left[\left(\mathbf{s}^{H} \mathbf{s}\right)^{-1} \mathbf{s}^{H} \mathbf{n}(t) / \mathbf{n}_{s^{\perp}}(t)\right]$ and the MVDR beamformer (17) takes the form:

$$
\begin{gathered}
\widehat{s}_{\mathrm{MVDR}}(t)=\mathbf{w}_{f}^{H} \mathbf{x}_{s}(t)-\left(\mathbf{s}^{H} \mathbf{s}\right)^{-1} \mathbf{s}^{H} \mathrm{E}\left[\mathbf{n}(t) / \mathbf{n}_{s^{\perp}}(t)\right] \\
=s(t)+\left(\mathbf{s}^{H} \mathbf{s}\right)^{-1} \mathbf{s}^{H}\left(\mathbf{n}(t)-\mathrm{E}\left[\mathbf{n}(t) / \mathbf{n}_{s^{\perp}}(t)\right]\right) .
\end{gathered}
$$

Expression (18) describes the output of the MVDR beamformer which takes only into account the distribution of the total noise $\mathbf{n}(t)$, and thus its potential non-Gaussianity and non-circularity in particular. Choosing $\mathbf{w}_{f}=\left(\mathbf{s}^{H} \mathbf{s}\right)^{-1} \mathbf{s}$ and comparing (18) and (15), we deduce that $\widehat{s}_{\mathrm{MMSE}}(t)$ and $\widehat{s}_{\mathrm{MVDR}}(t)$ have similar forms but where $\mathrm{E}[\mathbf{n}(t) / \mathbf{x}(t)]$ in (15), which contains information about the SOI distribution, has been replaced by $\mathrm{E}\left[\mathbf{n}(t) / \mathbf{n}_{s^{\perp}}(t)\right]$ in (18), which contains no information about the SOI distribution. If $\mathbf{n}(t)$ is Gaussian and circular, $\mathrm{E}\left[\mathbf{n}(t) / \mathbf{n}_{s} \perp(t)\right]$ is a linear function of $\mathbf{n}_{s}(t)$ and the MVDR beamformer (18) is a linear function of $\mathbf{x}(t)$ corresponding to the Capon beamformer [2]. If $\mathbf{n}(t)$ is Gaussian and non-circular, $\mathrm{E}\left[\mathbf{n}(t) / \mathbf{n}_{s^{\perp}}(t)\right]$ is a WL function of $\mathbf{n}_{s \perp}(t)$ and the MVDR beamformer (18) is a WL function of $\mathbf{x}(t)$ corresponding to the WL $\mathrm{MVDR}_{1}$ beamformer introduced in [9]. If $\mathbf{n}(t)$ is nonGaussian, $\mathrm{E}\left[\mathbf{n}(t) / \mathbf{n}_{s^{\perp}}(t)\right]$ is a non-linear function of $\mathbf{n}_{s^{\perp}}(t)$ and the MVDR beamformer (18) is a non-linear function of $\mathbf{x}(t)$ depending on the probability distribution of the total noise, unknown in practice. For this reason, we propose to approximate each component of $\mathrm{E}\left[\mathbf{n}(t) / \mathbf{n}_{s^{\perp}}(t)\right]$ by polynomial functions of the components of $\mathbf{n}_{s^{\perp}}(t)$ through the implementation of MVDR complex Volterra beamformers.

3) Complex Volterra beamformers: The general input/output relation of a full $M$ th-order complex Volterra beamformer is defined by [28] [29]:

$$
y(t)=w_{0}+\sum_{m=1}^{M} \sum_{q=0}^{m} \mathbf{w}_{m, q}^{H}\left[\mathbf{x}(t)^{\otimes(m-q)} \otimes \mathbf{x}^{*}(t)^{\otimes q}\right],
$$

where $\mathbf{w}_{m, q}$ with $0 \leq q \leq m$ and $1 \leq m \leq M$, is an $N^{m} \times 1$ complex filter and $w_{0}$ is a scalar which generally ensures that $y(t)$ is zero-mean whatever the entries. (19) defines, for $w_{0}=0$, a WL beamformer for $M=1$ and, whatever $w_{0}$, a full complex linear-quadratic beamformer [38] for $M=2$.

Let us recall that $\mathrm{E}\left[\mathbf{n}(t) / \mathbf{n}_{s^{\perp}}(t)\right]$ is the orthogonal projection of $\mathbf{n}(t)$ on the space of random vectors which are functions of $\mathbf{n}_{s^{\perp}}(t)$. Hence, the best $M$ th-order polynomial approximation of $\mathrm{E}\left[\mathbf{n}(t) / \mathbf{n}_{s^{\perp}}(t)\right]$ corresponds to the orthogonal projection of $\mathbf{n}(t)$ on the space generated by the components of (19) with $\mathbf{n}_{s^{\perp}}(t)$ instead of $\mathbf{x}(t)$. But the term $\mathbf{w}_{m, q}^{H}\left[\mathbf{n}_{s \perp}(t)^{\otimes(m-q)} \otimes \mathbf{n}_{s^{\perp}}^{*}(t)^{\otimes q}\right]$ brings information only if $\mathbf{w}_{m, q}^{H}\left[\mathbf{n}(t)^{\otimes(m-q)} \otimes \mathbf{n}^{*}(t)^{\otimes q}\right]$ is correlated with $\mathbf{n}(t)$, which requires that the $(m+1)$ th-order moments $\mathrm{E}\left[n_{i_{1}}(t) \ldots n_{i_{m-q}}(t) n_{i_{m-q+1}}^{*}(t) \ldots n_{i_{m+1}}^{*}(t)\right]$ are not zero, where $n_{i}(t)$ is the component $i$ of $\mathbf{n}(t)$. When the probability distribution of $\mathbf{n}(t)$ is symmetric, which is often the case in practice, the odd order moments of $\mathbf{n}(t)$ are zero. Hence, only $M$ th-order complex Volterra beamformers such that $M$ is odd and containing only polynomial terms of odd order are in general interesting for MVDR beamforming. 
For this reason, we limit the analysis in the following to third-order Volterra beamformers containing no SO terms and we compute and analyze the properties and performance of third-order Volterra MVDR beamformers exploiting only the statistics of the total noise.

\section{THIRD-ORDER VOLTERRA MVDR BEAMFORMERS}

\section{A. Third-order Volterra beamformers}

We consider a third-order Volterra beamformer whose output is defined by:

$$
\begin{aligned}
& y(t)=\mathbf{w}_{1,0}^{H} \mathbf{x}(t)+\mathbf{w}_{1,1}^{H} \mathbf{x}^{*}(t) \\
& +\mathbf{w}_{3,0}^{H}[\mathbf{x}(t) \otimes \mathbf{x}(t) \otimes \mathbf{x}(t)]+\mathbf{w}_{3,1}^{H}\left[\mathbf{x}(t) \otimes \mathbf{x}(t) \otimes \mathbf{x}^{*}(t)\right] \\
& +\mathbf{w}_{3,2}^{H}\left[\mathbf{x}(t) \otimes \mathbf{x}^{*}(t) \otimes \mathbf{x}^{*}(t)\right]+\mathbf{w}_{3,3}^{H}\left[\mathbf{x}^{*}(t) \otimes \mathbf{x}^{*}(t) \otimes \mathbf{x}^{*}(t)\right] \\
& \stackrel{\text { def }}{=} \widetilde{\mathbf{w}}{ }^{H} \widetilde{\mathbf{x}}(t) .
\end{aligned}
$$

Here $\mathbf{w}_{1,0}$ and $\mathbf{w}_{1,1}$ are $N \times 1$ spatial filters, $\mathbf{w}_{3,0}$, $\mathbf{w}_{3,1}, \mathbf{w}_{3,2}$ and $\mathbf{w}_{3,3}$ are $N^{3} \times 1$ spatial filters, and $\widetilde{\mathbf{x}}(t)$ and $\widetilde{\mathbf{w}}$ are $\left(2 N+4 N^{3}\right) \times 1$ vectors corresponding to the extended observation vector $\left[\mathbf{x}^{T}(t), \mathbf{x}^{H}(t),[\mathbf{x}(t) \otimes\right.$ $\mathbf{x}(t) \otimes \mathbf{x}(t)]^{T},\left[\mathbf{x}(t) \otimes \mathbf{x}(t) \otimes \mathbf{x}^{*}(t)\right]^{T},\left[\mathbf{x}(t) \otimes \mathbf{x}^{*}(t) \otimes\right.$ $\left.\left.\mathbf{x}^{*}(t)\right]^{T},\left[\mathbf{x}^{*}(t) \otimes \mathbf{x}^{*}(t) \otimes \mathbf{x}^{*}(t)\right]^{T}\right]^{T}$ and the extended spatial filter, $\left[\mathbf{w}_{1,0}^{T}, \mathbf{w}_{1,1}^{T}, \mathbf{w}_{3,0}^{T}, \mathbf{w}_{3,1}^{T}, \mathbf{w}_{3,2}^{T}, \mathbf{w}_{3,3}^{T}\right]^{T}$, respectively. The first-order terms describe the output of a linear beamformer when $\mathbf{w}_{1,1}=\mathbf{0}$ and of a WL beamformer otherwise. The third-order terms are called cubic (C) terms in the following and the number $r$ of $N^{3} \times 1$ cubic observation vectors, $\mathbf{x}_{3, q}(t) \stackrel{\text { def }}{=}\left[\mathbf{x}(t)^{\otimes(3-q)} \otimes \mathbf{x}^{*}(t)^{\otimes q}\right]$, appearing in (20) may be such that $0 \leq r \leq 4$, depending on the number of non-zero spatial filters $\mathbf{w}_{3, q},(0 \leq q \leq 3)$ giving rise to several particular third-order beamformers. The integer $q$ is called the index of $\mathbf{x}_{3, q}(t)$ and $\mathbf{w}_{3, q}$. In the presence of $r$ cubic terms $(1 \leq r \leq 4)$ ) having the index $q_{j}(1 \leq j \leq r)$, $\left(0 \leq q_{j} \leq 3\right)$, the beamformer (20) is called $\mathrm{L}-\mathrm{C}\left(q_{1}, q_{2}, . ., q_{r}\right)$ if the first order part is linear or WL-C $\left(q_{1}, q_{2}, . ., q_{r}\right)$ if the first order part is WL. In particular, when all the terms of (20) are considered, the associated beamformer is called WL-C $(0,1,2,3)$, whereas only L-C(1) beamformers have been considered in [39]. In fact we will show in the following sections that all the L-C $\left(q_{1}, q_{2}, . ., q_{r}\right)$ and WL-C $\left(q_{1}, q_{2}, . ., q_{r}\right)$ beamformers, $\left(0 \leq q_{j} \leq 3\right),(1 \leq j \leq r \leq 4)$, except the L-C(1) beamformer, exploit some informations about the potential HO non-circularity of the data and may be called third-order widely non linear beamformers, as suggested in [45].

\section{B. Third-order Volterra MVDR beamformers}

Using (1) into (20), we deduce from Section II-C2 that to build third-order Volterra MVDR beamformers exploiting the statistics of the total noise $\mathbf{n}(t)$ only, it is necessary to impose a constraint of non distortion of the SOI at the output (20) by keeping the term proportional to $s(t)$ and by nulling the non-linear terms of $s(t)$. The spatial filters of the first order terms must then verify the following constraints:

$$
\mathbf{w}_{1,0}^{H} \mathbf{s}=1 \text { and } \mathbf{w}_{1,1}^{H} \mathbf{s}^{*}=0,
$$

which correspond to the constraints imposed in [9] for WL $\mathrm{MVDR}_{1}$ beamforming. However, for the spatial filters of the cubic terms, the constraints of nulling the non-linear terms of $s(t)$ are not so obvious to obtain since $\mathbf{n}(t)$ is random. For this reason, as $\mathbf{s}$ is assumed to be known, it is possible to build an orthonormal basis $\left(\mathbf{u}_{1}, \ldots \mathbf{u}_{N-1}\right)$ of the space $\operatorname{span}(\mathbf{s})^{\perp}$, which means that $\left(\mathbf{s}, \mathbf{u}_{1}, \ldots \mathbf{u}_{N-1}\right)$ becomes an orthogonal basis of $\mathbb{C}^{N}$. The vector $\mathbf{n}(t)$ is then a linear combination of these vectors such that:

$$
\mathbf{n}(t)=\nu_{0}(t) \mathbf{s}+\sum_{i=1}^{N-1} \nu_{i}(t) \mathbf{u}_{i}
$$

where $\nu_{i}(t)(0 \leq i \leq N-1)$ are zero-mean a priori correlated (but uncorrelated with $s(t)$ ) random variables. For $0 \leq q \leq 3$, let us now insert (1) and (22) into the cubic term $\mathbf{w}_{3, q}^{H} \mathbf{x}_{3, q}(t)$ of (20). To cancel in this term, the non-linear terms of $s(t)$, it is equivalent to cancel all the terms of $\mathbf{w}_{3, q}^{H} \mathbf{x}_{3, q}(t)$ excluding the terms containing the $\mathbf{u}_{i}$ 's only. For example, for $q=1$, we must impose the $1+3(N-1)+3(N-1)^{2}=N^{3}-(N-1)^{3}$ following constraints:

$$
\begin{aligned}
\mathbf{w}_{3,1}^{H}\left(\mathbf{s} \otimes \mathbf{s} \otimes \mathbf{s}^{*}\right) & =0 \\
\mathbf{w}_{3,1}^{H}\left(\mathbf{u}_{i} \otimes \mathbf{s} \otimes \mathbf{s}^{*}\right) & =0, \mathbf{w}_{3,1}^{H}\left(\mathbf{s} \otimes \mathbf{u}_{i} \otimes \mathbf{s}^{*}\right)=0, \\
\mathbf{w}_{3,1}^{H}\left(\mathbf{s} \otimes \mathbf{s} \otimes \mathbf{u}_{i}^{*}\right) & =0, \quad 1 \leq i \leq N-1 \\
\mathbf{w}_{3,1}^{H}\left(\mathbf{u}_{i} \otimes \mathbf{u}_{j} \otimes \mathbf{s}^{*}\right) & =0, \mathbf{w}_{3,1}^{H}\left(\mathbf{u}_{i} \otimes \mathbf{s} \otimes \mathbf{u}_{j}^{*}\right)=0, \\
\mathbf{w}_{3,1}^{H}\left(\mathbf{s} \otimes \mathbf{u}_{i} \otimes \mathbf{u}_{j}^{*}\right) & =0, \quad 1 \leq i, j \leq N-1 .
\end{aligned}
$$

If we arrange the $N^{3}-(N-1)^{3}$ constraint vectors $\mathbf{s} \otimes \mathbf{s} \otimes \mathbf{s}^{*}$, $\mathbf{u}_{i} \otimes \mathbf{s} \otimes \mathbf{s}^{*}, \ldots ., \mathbf{s} \otimes \mathbf{u}_{i} \otimes \mathbf{u}_{j}^{*}, 1 \leq i, j \leq N-1$ appearing in (23) in a $N^{3} \times\left(N^{3}-(N-1)^{3}\right)$ matrix, denoted by $\mathbf{C}_{1}$, the set of constraints (23) can be written in a compact form given by $\mathbf{C}_{1}^{H} \mathbf{w}_{3,1}=\mathbf{0}_{N^{3}-(N-1)^{3}}$. For other values of $q(q=0,2,3)$, the constraints are still given by (23) but where $\mathbf{w}_{3,1}$ is replaced by $\mathbf{w}_{3, q}$ and where the $N^{3}-(N-1)^{3}$ constraint vectors contain now $q$ conjugate terms instead of 1. Again, for each value of $q$, if we arrange the associated $N^{3}-(N-1)^{3}$ constraint vectors in a $N^{3} \times\left(N^{3}-(N-1)^{3}\right)$ matrix, denoted by $\mathbf{C}_{q}$, the associated set of constraints can be written in a compact form given by:

$$
\mathbf{C}_{q}^{H} \mathbf{w}_{3, q}=\mathbf{0}_{N^{3}-(N-1)^{3}}, \quad q=0, . ., 3 .
$$

Let us now arrange the $2+4\left[N^{3}-(N-1)^{3}\right]$ constraint vectors appearing in (21) and (24) in a $\left(2 N+4 N^{3}\right) \times(2+$ $\left.4\left[N^{3}-(N-1)^{3}\right]\right)$ matrix $\mathbf{C}$ defined by

$$
\mathbf{C}=\operatorname{Diag}\left(\mathbf{s}, \mathbf{s}^{*}, \mathbf{C}_{0}, \mathbf{C}_{1}, \mathbf{C}_{2}, \mathbf{C}_{3}\right) \text {. }
$$

The global set of constraints takes the form

$$
\mathbf{C}^{H} \widetilde{\mathbf{w}}=\mathbf{f} \text {, }
$$

where $\mathbf{f}$ is the $\left(2+4\left[N^{3}-(N-1)^{3}\right]\right) \times 1$ vector defined by $\mathbf{f} \stackrel{\text { def }}{=}\left(1, \mathbf{0}_{1+4\left[N^{3}-(N-1)^{3}\right]}^{T}\right)^{T}$. Under the vector constraint (26) and using (1), the output (20) of the third-order Volterra 
beamformers takes the form

$$
\begin{aligned}
& y(t)=s(t)+\mathbf{w}_{1,0}^{H} \mathbf{n}(t)+\mathbf{w}_{1,1}^{H} \mathbf{n}^{*}(t) \\
& +\mathbf{w}_{3,0}^{H}[\mathbf{n}(t) \otimes \mathbf{n}(t) \otimes \mathbf{n}(t)]+\mathbf{w}_{3,1}^{H}\left[\mathbf{n}(t) \otimes \mathbf{n}(t) \otimes \mathbf{n}^{*}(t)\right] \\
& +\mathbf{w}_{3,2}^{H}\left[\mathbf{n}(t) \otimes \mathbf{n}^{*}(t) \otimes \mathbf{n}^{*}(t)\right]+\mathbf{w}_{3,3}^{H}\left[\mathbf{n}^{*}(t) \otimes \mathbf{n}^{*}(t) \otimes \mathbf{n}^{*}(t)\right] \\
& \stackrel{\text { def }}{=} s(t)+\widetilde{\mathbf{w}}^{H} \widetilde{\mathbf{n}}(t),
\end{aligned}
$$

where $\widetilde{\mathbf{n}}(t)$ is defined as $\widetilde{\mathbf{x}}(t)$ but with $\mathbf{n}(t)$ instead of $\mathbf{x}(t)$. The best SO estimate (20) or (27) of the SOI $s(t)$ exploiting the noise statistics only, thus corresponds to the output of the third-order Volterra beamformer $\widetilde{\mathbf{w}}_{\mathrm{MVDR}}$ which minimizes the time-averaged output power

$$
\widetilde{\mathbf{w}}^{H} \mathbf{R}_{\tilde{x}} \widetilde{\mathbf{w}}=\pi_{s}+\widetilde{\mathbf{w}}^{H} \mathbf{R}_{\tilde{n}} \widetilde{\mathbf{w}},
$$

under the vector constraint (26), where $\pi_{s} \stackrel{\text { def }}{=}<$ $\mathrm{E}|s(t)|^{2}>$, and $\mathbf{R}_{\widetilde{x}} \stackrel{\text { def }}{=}<\mathrm{E}\left[\widetilde{\mathbf{x}}(t) \widetilde{\mathbf{x}}^{H}(t)\right]>$ and $\mathbf{R}_{\widetilde{n}} \stackrel{\text { def }}{=}<$ $\mathrm{E}\left[\widetilde{\mathbf{n}}(t) \widetilde{\mathbf{n}}^{H}(t)\right]>$ are the time-averaged correlation matrix of $\widetilde{\mathbf{x}}(t)$ and $\widetilde{\mathbf{n}}(t)$, respectively:

$$
\widetilde{\mathbf{w}}_{\text {MVDR }} \stackrel{\text { def }}{=} \arg \left\{\min _{\mathbf{C}^{H} \widetilde{\mathbf{w}}=\mathbf{f}} \widetilde{\mathbf{w}}^{H} \mathbf{R}_{\widetilde{x}} \widetilde{\mathbf{w}}\right\} .
$$

Note that the minimization (29) can be efficiently numerically solvable by modern solvers, but, for performance analysis and implementation perspectives, we are interested to closed-form expressions of solutions. However, as $\widetilde{\mathbf{x}}(t)$ has redundant components for $N>1, \mathbf{R}_{\widetilde{x}}$ is singular for $N>1$ and the closed-form expressions of the solutions to (29) are difficult to derive (see e.g., [47, sec.19.3c]). To solve this problem, the redundancies of $\widetilde{\mathbf{x}}(t)$ must be removed and the constraints must be readjusted accordingly. But this task is not easy because the positions of the redundancies in $\widetilde{\mathbf{x}}(t)$ and in the constraint vectors introduced in (23) are different. But hopefully, this constrained optimization problem can be transformed to an unconstrained one, by using the equivalent Volterra GSC structure introduced in the following Subsection III-C for which the redundancies of the observations can be easily withdrawn.

When all the first and third order terms appearing in (20) are considered, (27) shows that the third-order Volterra MVDR beamformer exploits all the SO, FO and SIO circular and non-circular statistics of the total noise appearing in $\mathbf{R}_{\tilde{n}}$. However, if some terms in (20) are removed, some statistics are no longer exploited by the associated MVDR beamformers. For example the beamformer L-C(1) of [39] exploits only the SO, FO and SIO circular statistics of the total noise $\mathbf{n}(t)$ and thus does not exploit the potential noncircularity of the total noise. The exploitation of the SO non-circularity of $\mathbf{n}(t)$ is ensured if the first-order part of (20) is WL. Moreover, every WL-Cubic [resp., L-Cubic] beamformers with at least one [resp., two] third-order term in (20), exploit at least some FO non-circular statistics of $\mathbf{n}(t)$. Finally, the exploitation of the SIO non-circular statistics of $\mathbf{n}(t)$ by a third-order MVDR beamformer requires the presence of at least two third-order terms in (20), and thus cannot be done without exploiting also some FO noncircular statistics of $\mathbf{n}(t)$.

\section{Equivalent third-order Volterra GSC structure}

We show in this section that the third-order Volterra MVDR beamformers have equivalent third-order Volterra GSC structures. The GSC structure essentially transforms the constrained optimization problem (29) to an unconstrained one. This allows us to easily remove the redundancies of $\widetilde{\mathbf{x}}(t)$ and permits both the analytical computation of the third-order Volterra MVDR beamformers and much simpler adaptive implementations.

Any filter $\widetilde{\mathbf{w}}$ may be decomposed into two components:

$$
\widetilde{\mathbf{w}}=\widetilde{\mathbf{w}}_{f}-\widetilde{\mathbf{v}}
$$

where $\widetilde{\mathbf{w}}_{f} \stackrel{\text { def }}{=}\left[\mathbf{w}_{f}^{T}, \mathbf{0}_{N+4 N^{3}}^{T}\right]^{T}$, such that $\mathbf{w}_{f}$ is an $N \times 1$ filter satisfying $\mathbf{w}_{f}^{H} \mathbf{s}=1$ (e.g., $\left.\mathbf{w}_{f}=\|\mathbf{s}\|^{-2} \mathbf{s}\right)$. Since $\widetilde{\mathbf{w}}_{f}$ satisfies the constraint (26), the latter is equivalent to the constraint:

$$
\mathbf{C}^{H} \widetilde{\mathbf{v}}=\mathbf{0}_{2+4\left[N^{3}-(N-1)^{3}\right]},
$$

which means that $\widetilde{\mathbf{v}} \in \operatorname{span}(\mathbf{C})^{\perp}$, the space orthogonal to the columns of $\mathbf{C}$. Now consider a $\left[2 N+4 N^{3}\right] \times[2(N-$ 1) $\left.\left.+4(N-1)^{3}\right)\right]$ full column rank blocking matrix $\mathbf{B}$, whose columns span $\operatorname{span}(\mathbf{C})^{\perp}$. We obtain

$$
\mathbf{B}^{H} \mathbf{C}=\mathbf{O}_{\left[2(N-1)+4(N-1)^{3}\right] \times\left[2+4\left(N^{3}-(N-1)^{3}\right)\right]},
$$

and we deduce from (31) that $\widetilde{\mathbf{v}}$ is a linear combination of the columns of $\mathbf{B}$, which means that there exists a $[2(N-$ 1) $\left.+4(N-1)^{3}\right] \times 1$ filter $\widetilde{\mathbf{w}}_{a}$, such that

$$
\widetilde{\mathbf{v}}=\mathbf{B} \widetilde{\mathbf{w}}_{a} \text {. }
$$

Let us consider a $N \times(N-1)$ full rank matrix $\mathbf{B}_{1,0}$ verifying $\mathbf{B}_{1,0}^{H} \mathbf{s}=\mathbf{0}_{N-1}$. The matrix $\mathbf{B}_{1,0}=\left[\mathbf{u}_{1}, \ldots, \mathbf{u}_{N-1}\right]$ is such a solution. Using properties of the Kronecker product, it is straightforward to verify that the following matrix:

$$
\mathbf{B}=\operatorname{Diag}\left(\mathbf{B}_{1,0}, \mathbf{B}_{1,0}^{*}, \mathbf{B}_{3,0}, \mathbf{B}_{3,1}, \mathbf{B}_{3,2}, \mathbf{B}_{3,3}\right),
$$

is a full rank blocking matrix verifying (32), where $\mathbf{B}_{3, q}=$ $\left[\mathbf{B}_{1,0}^{\otimes(3-q)} \otimes \mathbf{B}_{1,0}^{*} \otimes q\right], q=0, . ., 3$. In this context, the MVDR filter (29) which satisfies the constraint (26) also corresponds to the filter $\widetilde{\mathbf{w}}=\widetilde{\mathbf{w}}_{f}-\mathbf{B} \widetilde{\mathbf{w}}_{a, \text { opt }}$, where $\mathbf{B}$ is defined by (34), $\widetilde{\mathbf{w}}_{f}$ is such that $\widetilde{\mathbf{w}}_{f}^{H} \widetilde{\mathbf{x}}(t)=\mathbf{w}_{f}^{H} \mathbf{x}(t)$ with $\mathbf{w}_{f}^{H} \mathbf{s}=1$ and $\widetilde{\mathbf{w}}_{a}$,opt minimizes the temporal mean of the power of $y(t)=\left(\widetilde{\mathbf{w}}_{f}-\mathbf{B} \widetilde{\mathbf{w}}_{a}\right)^{H} \widetilde{\mathbf{x}}(t):$

$$
\widetilde{\mathbf{w}}_{a, \mathrm{opt}}=\arg \left\{\min _{\widetilde{\mathbf{w}}_{a}}<\mathrm{E}\left|\left(\widetilde{\mathbf{w}}_{f}-\mathbf{B} \widetilde{\mathbf{w}}_{a}\right)^{H} \widetilde{\mathbf{x}}(t)\right|^{2}>\right\} .
$$

Denoting by $\mathbf{z}(t)$ the $(N-1) \times 1$ vector $\mathbf{z}(t) \stackrel{\text { def }}{=}$ $\mathbf{B}_{1,0}^{H} \mathbf{x}(t)=\mathbf{B}_{1,0}^{H} \mathbf{n}(t)$ and using the property that $\mathbf{B}_{3, q}^{H} \mathbf{x}_{3, q}(t) \stackrel{\text { def }}{=}\left[\mathbf{B}_{1,0}^{\otimes(3-q)} \otimes \mathbf{B}_{1,0}^{*} \otimes q\right]^{H}\left[\mathbf{x}(t)^{\otimes(3-q)} \otimes\right.$ $\left.\mathbf{x}^{*}(t)^{\otimes q}\right]=\left[\left(\mathbf{B}_{1,0}^{H} \mathbf{x}(t)\right)^{\otimes(3-q)} \otimes\left(\mathbf{B}_{1,0}^{T} \mathbf{x}^{*}(t)\right)^{\otimes q}\right]=$ $\left[\mathbf{z}(t)^{\otimes(3-q)} \otimes \mathbf{z}^{*}(t)^{\otimes q}\right]$, we obtain:

$$
\mathbf{B}^{H} \widetilde{\mathbf{x}}(t)=\mathbf{B}^{H} \widetilde{\mathbf{n}}(t)=\widetilde{\mathbf{z}}(t),
$$

where $\widetilde{\mathbf{z}}(t) \stackrel{\text { def }}{=}\left[\mathbf{z}^{T}(t), \mathbf{z}^{H}(t),[\mathbf{z}(t) \otimes \mathbf{z}(t) \otimes \mathbf{z}(t)]^{T},[\mathbf{z}(t) \otimes\right.$ $\left.\mathbf{z}(t) \otimes \mathbf{z}^{*}(t)\right]^{T},\left[\mathbf{z}(t) \otimes \mathbf{z}^{*}(t) \otimes \mathbf{z}^{*}(t)\right]^{T},\left[\mathbf{z}^{*}(t) \otimes \mathbf{z}^{*}(t) \otimes \mathbf{z}^{*}(t)\right]^{T}$ and the minimization problem (35) becomes

$$
\widetilde{\mathbf{w}}_{a, \mathrm{opt}}=\arg \left\{\min _{\widetilde{\mathbf{w}}_{a}}<\mathrm{E}\left|\mathbf{w}_{f}^{H} \mathbf{x}(t)-\widetilde{\mathbf{w}}_{a}^{H} \widetilde{\mathbf{z}}(t)\right|^{2}>\right\},
$$


Nevertheless, for $N>2$, the components of the $(N-1)^{3} \times 1$ vector $\mathbf{z}_{3, q}(t) \stackrel{\text { def }}{=}\left[\mathbf{z}(t)^{\otimes(3-q)} \otimes \mathbf{z}^{*}(t)^{\otimes q}\right]$, $q=0, . ., 3$ appearing in $\widetilde{\mathbf{z}}(t)$, which are the quantities $z_{i_{1}}(t), . ., z_{i_{3-q}}(t), z_{i_{4-q}}^{*}(t), . ., z_{i_{3}}^{*}(t)\left(1 \leq i_{j} \leq N-1\right),(1 \leq$ $j \leq 3)$, have some redundancies. This generates a singular time-averaged correlation matrix, $\mathbf{R}_{\tilde{z}} \stackrel{\text { def }}{=}<\mathrm{E}\left[\widetilde{\mathbf{z}}(t) \widetilde{\mathbf{z}}^{H}(t)\right]>$ of $\widetilde{\mathbf{z}}(t)$ and this makes difficult to solve (37). The removing of the redundancies of vectors $\mathbf{z}_{3, q}(t)$ is then required to solve easily (37). The non-redundant components of $\mathbf{z}_{3, q}(t)$ are then the quantities $z_{i_{1}}(t), . ., z_{i_{3-q}}(t), z_{i_{4-q}}^{*}(t), . ., z_{i_{3}}^{*}(t)$, $\left(1 \leq i_{j} \leq N-1\right),(1 \leq j \leq 3)$ such that $i_{1} \leq . . \leq i_{3-q}$ and $i_{4-q} \leq . . \leq i_{3}$. If we call $N_{q}$, the number of nonredundant components of $\mathbf{z}_{3, q}(t)$, it is easy to prove that $N_{0}=N_{3}=\frac{\left(N^{2}-1\right) N}{6}$ and $N_{1}=N_{2}=\frac{(N-1)^{2} N}{2}$. For each value of $q, 0 \leq q \leq 3$, if $\mathbf{z}_{3, q}^{\prime}(t)$ denotes the $N_{q} \times 1$ non-redundant vector associated with $\mathbf{z}_{3, q}(t)$, there exists a $N_{q} \times(N-1)^{3}$ selection matrix $\mathbf{K}_{q}$, that selects the nonredundant components of $\mathbf{z}_{3, q}(t)$, such that

$$
\mathbf{z}_{3, q}^{\prime}(t)=\mathbf{K}_{q} \mathbf{z}_{3, q}(t), q=0, . ., 3 .
$$

Defining $\widetilde{\mathbf{z}}^{\prime}(t) \stackrel{\text { def }}{=}\left[\mathbf{z}^{T}(t), \mathbf{z}^{H}(t), \mathbf{z}_{3,0}^{\prime T}(t), \mathbf{z}_{3,1}^{\prime T}(t), \mathbf{z}_{3,2}^{\prime T}(t), \mathbf{z}_{3,3}^{\prime T}(t)\right]^{T}$, the $N_{z} \times 1$ non-redundant extended observation vector associated with $\widetilde{\mathbf{z}}(t)$, where $N_{z}=2(N-1)\left(2 N^{2}-N+3\right) / 3$, we can write:

$$
\widetilde{\mathbf{z}}^{\prime}(t)=\mathbf{K} \widetilde{\mathbf{z}}(t)=\mathbf{K B}^{H} \widetilde{\mathbf{x}}(t)=\mathbf{K} \mathbf{B}^{H} \widetilde{\mathbf{n}}(t),
$$

where $\mathbf{K}$ is the $N_{z} \times\left[2(N-1)+4(N-1)^{3}\right]$ selection matrix selecting the non-redundant components of $\widetilde{\mathbf{z}}(t)$, defined by

$$
\mathbf{K}=\operatorname{Diag}\left(\mathbf{I}_{N-1}, \mathbf{I}_{N-1}, \mathbf{K}_{0}, \mathbf{K}_{1}, \mathbf{K}_{2}, \mathbf{K}_{3}\right) .
$$

Removing the redundancies, the optimization problem (37) consists in finding the $N_{z} \times 1$ vector $\widetilde{\mathbf{w}}_{a, \text { opt }}^{\prime}$ such that

$$
\widetilde{\mathbf{w}}_{a, \text { opt }}^{\prime}=\arg \left\{\min _{\widetilde{\mathbf{w}}_{a}^{\prime}}<\mathrm{E}\left|\mathbf{w}_{f}^{H} \mathbf{x}(t)-\widetilde{\mathbf{w}}_{a}^{\prime}{ }^{H} \widetilde{\mathbf{z}}^{\prime}(t)\right|^{2}>\right\} .
$$

The solution to this problem is given by

$$
\begin{aligned}
\widetilde{\mathbf{w}}_{a, \text { opt }}^{\prime} & \stackrel{\text { def }}{=} \mathbf{R}_{\tilde{z}^{\prime}}^{-1} \mathbf{R}_{\tilde{z}^{\prime}, x} \mathbf{w}_{f}=\left[\mathbf{K B}^{H} \mathbf{R}_{\tilde{x}} \mathbf{B} \mathbf{K}^{H}\right]^{-1} \mathbf{K B}^{H} \mathbf{R}_{\tilde{x}} \widetilde{\mathbf{w}}_{f} \\
& =\left[\mathbf{K B}^{H} \mathbf{R}_{\tilde{n}} \mathbf{B} \mathbf{K}^{H}\right]^{-1} \mathbf{K B}^{H} \mathbf{R}_{\tilde{n}} \widetilde{\mathbf{w}}_{f},
\end{aligned}
$$

where $\mathbf{R}_{\tilde{z}^{\prime}} \stackrel{\text { def }}{=}<\mathrm{E}\left[\tilde{\mathbf{z}}^{\prime}(t) \tilde{\mathbf{z}}^{\prime} H(t)\right]>$ and $\mathbf{R}_{\tilde{z}^{\prime}, x} \stackrel{\text { def }}{=}<$ $\mathrm{E}\left[\tilde{\mathbf{z}}^{\prime}(t) \mathbf{x}^{H}(t)\right]>$. The output $y(t)$ of the GSC structure is then given by:

$$
\begin{aligned}
y(t) & =\mathbf{w}_{f}^{H} \mathbf{x}(t)-\widetilde{\mathbf{w}}_{a, \mathrm{opt}}^{\prime} \tilde{\mathbf{z}}^{\prime}(t) \\
& =s(t)+\mathbf{w}_{f}^{H} \mathbf{n}(t)-\widetilde{\mathbf{w}}_{a, \mathrm{opt}}^{\prime} \mathbf{K B}^{H} \widetilde{\mathbf{x}}(t) \\
& =s(t)+\left(\widetilde{\mathbf{w}}_{f}-\mathbf{B} \mathbf{K}^{H} \widetilde{\mathbf{w}}_{a, \mathrm{opt}}^{\prime}\right)^{H} \widetilde{\mathbf{n}}(t) .
\end{aligned}
$$

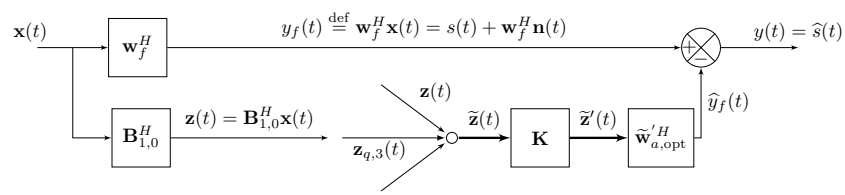

Fig.1 Equivalent third-order Volterra GSC structure.

The equivalent third-order Volterra GSC structure is depicted at Fig.1. This structure is not really surprising since it is a third-order Volterra approximation of the optimal GSC structure described by (18). Finally, note that equivalent third-order Volterra GSC structures can be similarly deduced for arbitrary $\mathrm{L}-\mathrm{C}\left(q_{1}, q_{2}, . ., q_{r}\right)$ and $\operatorname{WL}-\mathrm{C}\left(q_{1}, q_{2}, . ., q_{r}\right)$, $r=1, . ., 4$ MVDR beamformers, by defining the blocking and selection matrices $\mathbf{B}$ and $\mathbf{K}$ defined in (34) and (40), respectively, by $\operatorname{Diag}\left(\mathbf{B}_{1,0}, \mathbf{B}_{3, q_{1}}, \mathbf{B}_{3, q_{2}}, . ., \mathbf{B}_{3, q_{r}}\right)$ and $\operatorname{Diag}\left(\mathbf{I}_{N-1}, \mathbf{K}_{q_{1}}, \mathbf{K}_{q_{2}}, \ldots, \mathbf{K}_{q_{r}}\right)$ in the first case and by $\operatorname{Diag}\left(\mathbf{B}_{1,0}, \mathbf{B}_{1,0}^{*}, \mathbf{B}_{3, q_{1}}, \mathbf{B}_{3, q_{2}}, . ., \mathbf{B}_{3, q_{r}}\right) \quad$ and $\operatorname{Diag}\left(\mathbf{I}_{N-1}, \mathbf{I}_{N-1}, \mathbf{K}_{q_{1}}, \mathbf{K}_{q_{2}}, . ., \mathbf{K}_{q_{r}}\right)$ in the second case. Furthermore, the GSC structure associated with the Capon and the WL-MVDR 1 [9] beamformers can be deduced from the structure of Fig.1 by keeping only the $\mathbf{z}(t)$ term and the $\left(\mathbf{z}(t), \mathbf{z}^{*}(t)\right)$ terms respectively, in $\widetilde{\mathbf{z}}(t)$ and $\widetilde{\mathbf{z}}^{\prime}(t)$.

\section{Output SINR}

Using (42) into the orthogonal decomposition (43), it is straightforward to compute the ratio of the time-averaged powers of the SOI and the total noise at the output $y(t)$ of the third-order Volterra MVDR beamformers, referred to as the output SINR. This output SINR is given by

$\operatorname{SINR}_{\mathrm{MVDR}}=$
$\mathbf{w}_{f}^{H}\left[\mathbf{R}_{n}-\mathbf{R}_{\tilde{n}, n}^{H} \mathbf{B K}^{H}\left(\mathbf{K B}^{H} \mathbf{R}_{\tilde{n}} \mathbf{B} \mathbf{K}^{H}\right)^{-1} \mathbf{K B}^{H} \mathbf{R}_{\tilde{n}, n}\right] \mathbf{w}_{f}$

where $\mathbf{R}_{\tilde{n}, n} \stackrel{\text { def }}{=}<\mathrm{E}\left[\widetilde{\mathbf{n}}(t) \mathbf{n}^{H}(t)\right]>$. In the particular case $N=2, \mathbf{z}(t)$ is scalar-valued, $\widetilde{\mathbf{z}}(t)$ has no redundancy, $\mathbf{K}=\mathbf{I}$ and (44) reduces to

$$
\mathrm{SINR}_{\mathrm{MVDR}}=\frac{\pi_{s}}{\mathbf{w}_{f}^{H}\left[\mathbf{R}_{n}-\mathbf{R}_{\tilde{n}, n}^{H} \mathbf{B}\left(\mathbf{B}^{H} \mathbf{R}_{\tilde{n}} \mathbf{B}\right)^{-1} \mathbf{B}^{H} \mathbf{R}_{\tilde{n}, n}\right] \mathbf{w}_{f}}
$$

Note that although the couple $\left(\mathbf{w}_{f}, \mathbf{B}_{1,0}\right)$ satisfying $\mathbf{w}_{f}^{H} \mathbf{s}=$ 1 and $\operatorname{span}\left(\mathbf{B}_{1,0}\right)=\operatorname{span}(\mathbf{s})^{\perp}$, is not unique, the SINR given by (44) and (45) does not depend on this choice. Moreover, denoting by $\mathrm{SINR}_{\mathrm{B}}$, the SINR at the output of the MVDR beamformer $\mathrm{B}$, the following inequalities can be deduced from the inclusion principle applied to the constrained minimization (29):

$$
\begin{aligned}
& \operatorname{SINR}_{\mathrm{L}} \leq \operatorname{SINR}_{\mathrm{L}-\mathrm{C}\left(q_{1}, . ., q_{r}\right)} \leq \operatorname{SINR}_{\mathrm{L}-\mathrm{C}\left(q_{1}, . ., q_{r}, q_{r+1}\right)} \\
& \operatorname{SINR}_{\mathrm{WL}} \leq \operatorname{SINR}_{\mathrm{WL}-\mathrm{C}\left(q_{1}, . ., q_{r}\right)} \leq \operatorname{SINR}_{\mathrm{WL}-\mathrm{C}\left(q_{1}, . ., q_{r}, q_{r+1}\right)} \\
& \operatorname{SINR}_{\mathrm{L}-\mathrm{C}\left(q_{1}, . ., q_{r}\right)} \leq \operatorname{SINR}_{\mathrm{WL}-\mathrm{C}\left(q_{1}, . ., q_{r}\right)}(46)
\end{aligned}
$$

for $0 \leq q_{j} \leq 3,1 \leq j \leq 4,1 \leq r \leq 3$, where $\operatorname{SINR}_{\mathrm{L}}$ and $\mathrm{SINR}_{\mathrm{WL}}$, such that $\mathrm{SINR}_{\mathrm{L}} \leq \mathrm{SINR}_{\mathrm{WL}}$ correspond to the SINR at the output of the Capon and WL MVDR 1 [9] beamformer, respectively such that

$$
\operatorname{SINR}_{\mathrm{L}}=\pi_{s} \mathbf{s}^{H} \mathbf{R}_{n}^{-1} \mathbf{s} .
$$

\section{PERFormance IN THE PRESENCE OF ONE INTERFERENCE}

In this section, we analyze the performance of several third-order Volterra MVDR beamformers in the presence of one non-Gaussian and potentially non-circular interference. 


\section{A. Total noise model}

We consider the observation model (1) where $\mathbf{n}(t)$ is assumed to contain the contribution of one interference and a background noise, both statistically independent of $s(t)$. Under these assumptions, $\mathbf{n}(t)$ can be written as

$$
\mathbf{n}(t)=j(t) \mathbf{j}+\mathbf{n}_{G}(t)
$$

where $j(t)$ and $\mathbf{j}$ correspond to the complex envelope, assumed to be zero mean and potentially non-Gaussian and/or non-circular, and the steering vector of the interference, respectively, whereas $\mathbf{n}_{G}(t)$ is the background noise vector, assumed to be zero-mean, Gaussian, stationary, circular and spatially white, such that each component has a power $\eta_{2}$.

\section{B. Performance of $L-C(q) M V D R$ beamformers}

In this sub-section, we analyze from (45) and the results of Appendix explaining the way to compute (45) for the model (48), the performance of L-C $(q)$ MVDR beamformers, $q=0, . ., 3$ for one arbitrary interference.

1) SINR at the output of Capon, WL MVDR $R_{1}$ and $L-C(q)$ beamformers: For one interference, the SINR (47) at the output of the Capon's beamformer is simply given by [9]

$$
\mathrm{SINR}_{\mathrm{L}}=\epsilon_{s}\left(1-\frac{\epsilon_{j}}{1+\epsilon_{j}} \alpha^{2}\right) \text {. }
$$

Here, $\epsilon_{s}$ and $\epsilon_{j}$ are defined by $\epsilon_{s} \stackrel{\text { def }}{=}\|\mathbf{s}\|^{2} \pi_{s} / \eta_{2}$ and $\epsilon_{j} \stackrel{\text { def }}{=}$ $\|\mathbf{j}\|^{2} \pi_{j} / \eta_{2}$ where $\pi_{j} \stackrel{\text { def }}{=}<\mathrm{E}\left|j^{2}(t)\right|>$ is the input power of the interference received by an omnidirectional antenna and $\alpha$, such that $0 \leq \alpha \leq 1$, is the modulus of the spatial correlation coefficient between the interference and the SOI, defined by

$$
\alpha \stackrel{\text { def }}{=}\left|\mathbf{s}^{H} \mathbf{j}\right| /\|\mathbf{s}\|\|\mathbf{j}\| \text {. }
$$

Using these notations, it has been shown in [9] that the SINR at the output of the WL MVDR 1 beamformer is given by

$$
\begin{aligned}
& \operatorname{SINR}_{\mathrm{WL}}=\epsilon_{s} \\
& \frac{\left[1+\epsilon_{j}\left(1+\beta^{2}\right)+\epsilon_{j}^{2}\left(1-\left|\gamma_{j}\right|^{2}\right) \beta^{2}\right]^{2}-\epsilon_{j}^{2} \alpha^{4}\left|\gamma_{j}\right|^{2}}{\left[\left(1+\epsilon_{j}\right)^{2}-\epsilon_{j}^{2}\left|\gamma_{j}\right|^{2}\right]\left[1+\epsilon_{j}\left(1+\beta^{2}\right)+\epsilon_{j}^{2}\left(1-\left|\gamma_{j}\right|^{2}\right) \beta^{2}\right]}
\end{aligned}
$$

where $\gamma_{j}$ is defined by (3) with $j(t)$ instead of $u(t)$ and $\beta^{2} \stackrel{\text { def }}{=} 1-\alpha^{2}$.

To compute analytically the SINR at the output of the L-C $(q)$ MVDR beamformers, $q=0, . ., 3$, for an arbitrary interference, we assume that $N=2$ for which $\mathbf{z}(t)$ is scalarvalued. Based on a particular choice of $\left(\mathbf{w}_{f}, \mathbf{B}_{1,0}\right)$, we deduce from (45) and the results of Appendix, after tedious computations and for $q=0, . ., 3$, the following results:

$$
\operatorname{SINR}_{\mathrm{LC}(q)}=\epsilon_{s} \frac{\left(1+\epsilon_{j} \beta^{2}\right) A_{q}}{\left(1+\epsilon_{j}\right) A_{q}-\alpha^{2} \beta^{6} \epsilon_{j}^{4} D_{q}}
$$

where the quantities $A_{q}$ and $D_{q}, q=0, \ldots, 3$ are defined by

$$
\begin{aligned}
& A_{0}=A_{2} \stackrel{\text { def }}{=} \\
& \beta^{8} \epsilon_{j}^{4}\left(\chi_{j, c}-\left|\kappa_{j, n c, 2}^{2}\right|\right)+\beta^{6} \epsilon_{j}^{3}\left(\chi_{j, c}+9 \kappa_{j, c}-6 \operatorname{Re}\left(\gamma_{j} \kappa_{j, n c, 2}^{*}\right)\right)
\end{aligned}
$$

$$
\begin{aligned}
& +9 \beta^{4} \epsilon_{j}^{2}\left(\kappa_{j, c}+2-\left|\gamma_{j}^{2}\right|\right)+24 \beta^{2} \epsilon_{j}+6, \\
A_{1} & \stackrel{\text { def }}{=} \beta^{8} \epsilon_{j}^{4}\left(\chi_{j, c}-\kappa_{j, c}^{2}\right)+\beta^{6} \epsilon_{j}^{3}\left(\chi_{j, c}+\kappa_{j, c}\right) \\
& +\beta^{4} \epsilon_{j}^{2}\left(5 \kappa_{j, c}+2\right)+8 \beta^{2} \epsilon_{j}+2, \\
A_{3} \stackrel{\text { def }}{=} \beta^{8} \epsilon_{j}^{4}\left(\chi_{j, c}-\left|\kappa_{j, n c, 1}^{2}\right|\right)+\beta^{6} \epsilon_{j}^{3}\left(\chi_{j, c}+9 \kappa_{j, c}\right) & \quad+9 \beta^{4} \epsilon_{j}^{2}\left(\kappa_{j, c}+2\right)+24 \beta^{2} \epsilon_{j}+6, \\
& \quad \stackrel{\text { def }}{D_{0}}\left|\kappa_{j, n c, 2}-3 \gamma_{j}\right|^{2} ; D_{1} \stackrel{\text { def }}{=}\left(\kappa_{j, c}-2\right)^{2} ; \\
D_{2} & \stackrel{\text { def }}{=}\left|\left(\kappa_{j, n c, 2}-\gamma_{j}\right)+2 \gamma_{j} /\left(\beta^{2} \epsilon_{j}\right)\right|^{2} ; D_{3} \stackrel{\text { def }}{=} \mid \kappa_{j, n c, 1}^{2}
\end{aligned}
$$

Here the FO and SIO coefficients $\kappa_{j, c}, \kappa_{j, n c, 1}, \kappa_{j, n c, 2}$ and $\chi_{j, c}$ are defined by (2) and (4) with $j(t)$ instead of $u(t)$. Defining the SINR gain with respect to the Capon beamformer, $G_{\mathrm{B}} \stackrel{\text { def }}{=} \mathrm{SINR}_{\mathrm{B}} / \mathrm{SINR}_{\mathrm{L}}$, obtained in using the beamformer $\mathrm{B}$ instead of Capon beamformer, we derive from (49), (51) and (52) and for $q=0, . ., 3$ :

$$
\begin{aligned}
G_{\mathrm{WL}} & = \\
1+ & \frac{\alpha^{2} \beta^{2} \epsilon_{j}^{2}\left|\gamma_{j}\right|^{2}}{\left(1+\epsilon_{j} \beta^{2}\right)\left[1+\epsilon_{j}\left(1+\beta^{2}\right)+\epsilon_{j}^{2}\left(1-\left|\gamma_{j}\right|^{2}\right) \beta^{2}\right]} \\
G_{\mathrm{LC}(q)} & =1+\frac{\alpha^{2} \beta^{6} \epsilon_{j}^{4} D_{q}}{\left(1+\epsilon_{j}\right) A_{q}-\alpha^{2} \beta^{6} \epsilon_{j}^{4} D_{q}},
\end{aligned}
$$

Expressions (58) and (59) show that the WL and the L$\mathrm{C}(q), q=0, . ., 3$ MVDR beamformers bring no information with respect to the Capon beamformer (i.e., $G=1$ ), in the absence of interference $\left(\epsilon_{j}=0\right)$ or when the steering vectors of the SOI and interference are either orthogonal $(\alpha=0)$ or collinear $(\beta=0)$. In the first case, the Capon beamformer completely rejects the interference whereas in the second case, a spatial rejection is impossible. Otherwise, the SINR and the gain in SINR $G$, of the considered non-linear MVDR beamformers depend on both $\epsilon_{j}$, and on the interference to noise ratio (INR) per omnidirectional antenna (INR = $\left.\pi_{j} / \eta_{2}\right)$ in particular, and the statistical properties of the interference, and more precisely on the coefficients $\gamma_{j}, \kappa_{j, c}$, $\kappa_{j, n c, 1}, \kappa_{j, n c, 2}$ and $\chi_{j, c}$. In particular, for weak values of $\epsilon_{j}$ $\left(\epsilon_{j} \ll 1\right)$, (58) and (59) show that the WL and the L-C $(q)$, $q=0, . ., 3$ MVDR beamformers improve only very weakly the Capon beamformer since the latter is very powerful in this case $\left(\operatorname{SINR}_{\mathrm{L}} \approx \epsilon_{s}\right)$, as shown by (49). Nevertheless, as the interference becomes not too weak, the practical interest of the L-C $(q)(q=0, . ., 3)$ MVDR beamformers may appear, depending on the interference scenario, as it is discussed in the following sub-sections.

2) L-C(1) beamformer: The L-C(1) MVDR beamformer exploits the potential non-Gaussianity of the interference through the coefficients $\kappa_{j, c}$ and $\chi_{j, c}$. Expressions (56) and (59) for $q=1$ show that it brings no information with respect to the Capon beamformer if $\kappa_{j, c}=2$, which is satisfied by a circular Gaussian interference, for which the Capon beamformer is optimal. However, the L-C(1) MVDR beamformer improves the Capon beamformer provided that $\kappa_{j, c} \neq 2$, which generally occurs for a non Gaussian interference, circular or not, but also for a non-circular 
Gaussian interference for which $\kappa_{j, c}=2+\left|\gamma_{j}\right|^{2}$ (6). Thus, the L-C(1) MVDR beamformer also exploits the potential SO non-circularity of a Gaussian interference.

To simplify the analysis of the L-C(1) MVDR beamformer performance, we assume a strong interference $\left(\epsilon_{j} \gg\right.$ $1)$ in the following. Note that for an array with identical antennas, $\epsilon_{j} \gg 1$ means that INR $\gg 1 / N$, which does not necessarily require very large values of INR, depending on the value of $N$. For $N=2$, the strong assumption means INR $\gg 0.5$ whereas for $N=5$, it means INR $\gg 0.2$. The meaning of $\approx$ depends on the beamformer and on the $\alpha$ parameter in particular.

For $\kappa_{j, c} \neq 2$, assuming a strong interference $\left(\epsilon_{j} \gg 1\right)$, (54), (56) and (59) show that if $\chi_{j, c} \neq \kappa_{j, c}^{2}$, the L-C(1) MVDR beamformer improves only very marginally the Capon beamformer since $G_{\mathrm{LC}(1)}-1$ becomes inversely proportional to $\epsilon_{j}$ and thus very weak. Such a situation occurs (see (6) and (7)) for example for a non-circular Gaussian interference for which the L-C(1) MVDR beamformer remains less powerful than the WL MVDR beamformer. However, for a strong interference such that $\chi_{j, c}=\kappa_{j, c}^{2}$ and $\beta \neq 0$, (52) and (59) for $q=1$ become:

$$
\begin{aligned}
& \operatorname{SINR}_{\mathrm{LC}(1)} \approx \epsilon_{s}\left(1-\frac{\alpha^{2}\left(5 \kappa_{j, c}-4\right)}{\kappa_{j, c}\left(\kappa_{j, c}+1\right)-\alpha^{2}\left(\kappa_{j, c}-2\right)^{2}}\right)(60) \\
& G_{\mathrm{LC}(1)} \approx 1+\frac{\alpha^{2}\left(\kappa_{j, c}-2\right)^{2}}{\kappa_{j, c}\left(\kappa_{j, c}+1\right)-\alpha^{2}\left(\kappa_{j, c}-2\right)^{2}}, \\
& \chi_{j, c}=\kappa_{j, c}^{2}, \alpha \neq 1, \epsilon_{j} \gg 1 .(61)
\end{aligned}
$$

Note that the condition $\chi_{j, c}=\kappa_{j, c}^{2}$ means that $|j(t)|$ takes at most two values corresponding to zero and a non-zero constant value. In particular, for an interference such that $|j(t)|$ is constant (CPM, FSK or non-filtered PSK interference), $\chi_{j, c}=\kappa_{j, c}^{2}=1, \operatorname{SINR}_{\mathrm{LC}(1)} \approx 2 \epsilon_{s}\left[1-1 /\left(2-\alpha^{2}\right)\right]$ and $G_{\mathrm{LC}(1)} \approx 2 /\left(2-\alpha^{2}\right)$, which shows that $\operatorname{SINR}_{\mathrm{LC}(1)}$ decreases with $\alpha$, whereas $G_{\mathrm{LC}(1)}$ increases with $\alpha$ and is upper-bounded by 2 as $\alpha$ approaches unity. Moreover, as $\kappa_{j, c} \geq 1$, it is straightforward to prove that $\operatorname{SINR}_{\mathrm{LC}(1)}$ and $G_{\mathrm{LC}(1)}$ given by (60) and (61), respectively, first decrease from $\operatorname{SINR}_{\mathrm{LC}(1)} \approx \epsilon_{s}\left[1-\alpha^{2} /\left(2-\alpha^{2}\right)\right]$ and $G_{\mathrm{LC}(1)} \approx$ $1+\alpha^{2} /\left(2-\alpha^{2}\right)$ for $\kappa_{j, c}=1$ to $\operatorname{SINR}_{\mathrm{LC}(1)} \approx \epsilon_{s}\left(1-\alpha^{2}\right)$ and $G_{\mathrm{LC}(1)} \approx 1$ for $\kappa_{j, c}=2$ and then increase to $\mathrm{SINR}_{\mathrm{LC}(1)} \approx$ $\epsilon_{s}$ and $G_{\mathrm{LC}(1)} \approx 1+\alpha^{2} /\left(1-\alpha^{2}\right)$, for very high values of $\kappa_{j, c}$. In this latter case, $\operatorname{SINR}_{\mathrm{LC}(1)}$ is maximum and corresponds to the one without interference, the interference is completely cancelled by the L-C(1) MVDR beamformer whatever $\alpha(\alpha \neq 1)$, and the gain GL-C(1) infinitely increases with $\alpha(\alpha \neq 1)$. This shows that very efficient interference rejection and very high performance gain may be obtained in using the L-C(1) MVDR beamformer instead of the Capon beamformer, hence the great interest of the L$\mathrm{C}(1)$ beamformer even for $N=2$. Such a situation occurs in particular for an impulsive interference such that $|j(t)|$ is Bernoulli distributed. In this case, it has been shown in (11) that $\chi_{j, c}=\kappa_{j, c}^{2}=1 / p^{2}$ and both the SINR (60) and the performance gain (61) increase toward their maximum values $\operatorname{SINR}_{\mathrm{LC}(1)} \approx \epsilon_{s}$ and $G_{\mathrm{LC}(1)} \approx 1+\alpha^{2} /\left(1-\alpha^{2}\right)$, as $p$ decreases to zero, i.e., for very impulsive interference.

3) $L-C(q), q=0,2$ beamformers: The $\mathrm{L}-\mathrm{C}(q), q=0,2$ MVDR beamformers not only exploit the potential nonGaussiannity of the interference through the coefficients $\left(\kappa_{j, c}, \chi_{j, c}\right)$ but also the potential SO and FO non-circularity of the latter through the coefficients $\gamma_{j}$ and $\kappa_{j, n c, 2}$. Expressions (56), (57) and (59) for $q=0,2$ show that the L-C(0) and L-C(2) MVDR beamformers bring no information with respect to the Capon beamformer when $\kappa_{j, n c, 2}=3 \gamma_{j}$ and $\kappa_{j, n c, 2}=\gamma_{j}\left(1-2 /\left(\beta^{2} \epsilon_{j}\right)\right)$ respectively. This occurs in particular for a SO circular interference such that $\kappa_{j, n c, 2}=0$. Such an interference may correspond to a circular, a $2^{k}$-PSK $(k>1)$ or a square $4 M^{2}$-QAM interference. Moreover, for a non-circular Gaussian interference, for which $\kappa_{j, n c, 2}=3 \gamma_{j}$ (6), the L-C(0) MVDR beamformer improves no more the Capon beamformer contrary to the L-C(2) MVDR beamformer which may improve the latter. Nevertheless the L$\mathrm{C}(0)$ and L-C(2) MVDR beamformers generally improve the Capon beamformer when $\kappa_{j, n c, 2} \neq 3 \gamma_{j}$ and $\kappa_{j, n c, 2} \neq$ $\gamma_{j}\left(1-2 /\left(\beta^{2} \epsilon_{j}\right)\right)$ respectively. This requires a SO noncircular interference which is not Gaussian in the first case but which may be Gaussian in the second case. In these cases, assuming a strong interference, (53), (56), (57) and (59) show that if $\chi_{j, c} \neq\left|\kappa_{j, n c, 2}\right|^{2}$, the $\mathrm{L}-\mathrm{C}(0)$ and $\mathrm{L}$ C(2) MVDR beamformers improve only very marginally the Capon beamformer. Such a situation occurs for example for a strong non-circular Gaussian interference. However, for a strong interference such that $\chi_{j, c}=\left|\kappa_{j, n c, 2}\right|^{2}$ and $\beta \neq 0$, (52) and (59) for $q=0,2$ become:

$$
\mathrm{SINR}_{\mathrm{LC}(0)} \approx \epsilon_{s}
$$

$\left(1-\frac{9 \alpha^{2}\left(\kappa_{j, c}-\left|\gamma_{j}^{2}\right|\right)}{\left|\kappa_{j, n c, 2}\right|^{2}+9 \kappa_{j, c}-6 \operatorname{Re}\left(\gamma_{j} \kappa_{j, n c, 2}^{*}\right)-\alpha^{2}\left|\kappa_{j, n c, 2}-3 \gamma_{j}\right|^{2}}\right)(62$

$$
\mathrm{SINR}_{\mathrm{LC}(2)} \approx \epsilon_{s}
$$$$
\left(1-\frac{\alpha^{2}\left(9 \kappa_{j, c}-\left|\gamma_{j}^{2}\right|-4 \operatorname{Re}\left(\gamma_{j} \kappa_{j, n c, 2}^{*}\right)\right)}{\left|\kappa_{j, n c, 2}\right|^{2}+9 \kappa_{j, c}-6 \operatorname{Re}\left(\gamma_{j} \kappa_{j, n c, 2}^{*}\right)-\alpha^{2}\left|\kappa_{j, n c, 2}-\gamma_{j}\right|^{2}}\right)(63)
$$

$$
G_{\mathrm{LC}(0)} \approx
$$

$$
1+\frac{\alpha^{2}\left|\kappa_{j, n c, 2}-3 \gamma_{j}\right|^{2}}{\left|\kappa_{j, n c, 2}\right|^{2}+9 \kappa_{j, c}-6 \operatorname{Re}\left(\gamma_{j} \kappa_{j, n c, 2}^{*}\right)-\alpha^{2}\left|\kappa_{j, n c, 2}-3 \gamma_{j}\right|^{2}}
$$

$G_{\mathrm{LC}(2)} \approx$

$$
\begin{array}{r}
1+\frac{\alpha^{2}\left|\kappa_{j, n c, 2}-\gamma_{j}\right|^{2}}{\left|\kappa_{j, n c, 2}\right|^{2}+9 \kappa_{j, c}-6 \operatorname{Re}\left(\gamma_{j} \kappa_{j, n c, 2}^{*}\right)-\alpha^{2}\left|\kappa_{j, n c, 2}-\gamma_{j}\right|^{2}}, \\
\chi_{j, c}=\left|\kappa_{j, n c, 2}\right|^{2}, \quad \alpha \neq 1, \epsilon_{j} \gg 1 .
\end{array}
$$

The condition $\chi_{j, c}=\left|\kappa_{j, n c, 2}\right|^{2}$ means that $j^{2}(t)$ takes at most two values corresponding to zero and a non-zero constant value, and consequently $j(t)$ is necessarily rectilinear. In particular, for an interference such that $j^{2}(t)$ is constant (ex: non-filtered BPSK interference), $\chi_{j, c}=\left|\kappa_{j, n c, 2}\right|^{2}=1$, and we obtain:

$$
\operatorname{SINR}_{\mathrm{LC}(0)} \approx \epsilon_{s}, \operatorname{SINR}_{\mathrm{LC}(2)} \approx \epsilon_{s}\left(1-\alpha^{2}\right), G_{\mathrm{LC}(2)} \approx 1,
$$




$$
G_{\mathrm{LC}(0)} \approx 1+\frac{\alpha^{2}}{1-\alpha^{2}} ; \chi_{j, c}=\left|\kappa_{j, n c, 2}\right|^{2}=1, \alpha \neq 1, \epsilon_{j} \gg 1 .
$$

whereas (51) and (58) become

$$
\begin{aligned}
\operatorname{SINR}_{\mathrm{WL}} & \approx \epsilon\left(1-\frac{\alpha^{2}}{2-\alpha^{2}}\right) \\
G_{\mathrm{WL}} & \approx 1+\frac{\alpha^{2}}{2-\alpha^{2}}, \quad\left|\gamma_{j}\right|=1, \alpha \neq 1, \epsilon_{j} \gg 1 .
\end{aligned}
$$

In this case, (66) shows that the L-C(2) MVDR beamformer does not improve the Capon beamformer whereas (66)-(69) show that the L-C(0) MVDR beamformer outperforms both the Capon and the WL MVDR beamformer by completely canceling the interference whatever $\alpha(\alpha \neq 1)$ and the gain $G_{\mathrm{LC}(0)}$ infinitely increases with $\alpha(\alpha \neq 1)$. Such very high performance are also obtained at the output of both the L-C(0) and the L-C(2) MVDR beamformers when the rectilinear interference is very impulsive, such that $|j(t)|$ is Bernoulli distributed with a very small value of $p$. In this case, $\operatorname{SINR}_{\mathrm{LC}(0)} \approx \operatorname{SINR}_{\mathrm{LC}(2)} \approx \epsilon_{s}$ and $G_{\mathrm{LC}(0)} \approx G_{\mathrm{LC}(2)} \approx 1+\alpha^{2} /\left(1-\alpha^{2}\right)$, proving the great interest of these linear-Cubic beamformers.

4) L-C(3) beamformer: The L-C(3) MVDR beamformer exploits the potential non-Gaussiannity of the interference through the coefficients $\left(\kappa_{j, c}, \chi_{j, c}\right)$ and the potential FO non-circularity of the latter through the coefficients $\kappa_{j, n c, 1}$. We deduce from (55), (57) and (59) for $q=3$, that the LC(3) MVDR beamformer brings no information with respect to the Capon beamformer when $\kappa_{j, n c, 1}=0$. This occurs in particular for a FO circular interference such as, for example, a $2^{k}$-PSK $(k>2)$ interference. Nevertheless the L-C(3) MVDR beamformer generally improves the Capon beamformer when $\kappa_{j, n c, 1} \neq 0$. This is in particular the case for a non-circular Gaussian interference, for a rectilinear interference (BPSK, $M$-ASK, impulsive) but also for particular SO circular non-Gaussian interference such as QPSK or 16-QAM interference. In this case, assuming a strong interference, (55), (57) and (59) for $q=3$ show that if $\chi_{j, c} \neq\left|\kappa_{j, n c, 1}\right|^{2}$, the L-C(3) beamformer improves only very marginally the Capon beamformer. Such a situation occurs for example for a strong non-circular Gaussian interference or for $M$-ASK or 16-QAM interference. However, for a strong interference such that $\chi_{j, c}=\left|\kappa_{j, n c, 1}\right|^{2}$ and $\alpha \neq 1$, (52) and (59) for $q=3$ become:

$$
\begin{aligned}
\operatorname{SINR}_{\mathrm{LC}(3)} \approx & \epsilon_{s}\left(1-\frac{9 \alpha^{2} \kappa_{j, c}}{9 \kappa_{j, c}+\left(1-\alpha^{2}\right)\left|\kappa_{j, n c, 1}\right|^{2}}\right)(70) \\
G_{\mathrm{LC}(3)} \approx & 1+\frac{\alpha^{2}\left|\kappa_{j, n c, 1}\right|^{2}}{9 \kappa_{j, c}+\left(1-\alpha^{2}\right)\left|\kappa_{j, n c, 1}\right|^{2}}, \\
& \chi_{j, c}=\left|\kappa_{j, n c, 1}\right|^{2}, \alpha \neq 1, \epsilon_{j} \gg 1(71)
\end{aligned}
$$

The condition $\chi_{j, c}=\left|\kappa_{j, n c, 1}\right|^{2}$ means that $j^{4}(t)$ takes at most two values corresponding to zero and a non-zero constant value. In particular, for an interference such that $j^{4}(t)$ is constant (ex: non-filtered BPSK or QPSK interference), $\chi_{j, c}=\left|\kappa_{j, n c, 1}\right|^{2}=1$, and we obtain:

$$
\begin{gathered}
\operatorname{SINR}_{\mathrm{LC}(3)} \approx \epsilon_{s}\left(1-\frac{9 \alpha^{2}}{10-\alpha^{2}}\right) \\
G_{\mathrm{LC}(3)} \approx 1+\frac{\alpha^{2}}{10-\alpha^{2}} ; \chi_{j, c}=\left|\kappa_{j, n c, 1}\right|^{2}=1, \alpha \neq 1, \epsilon_{j} \gg 1,
\end{gathered}
$$

which proves that the L-C(3) MVDR beamformer improves slightly the Capon beamformer and remains less powerful than the WL beamformer for a rectilinear interference. Finally, for a very impulsive rectilinear interference such that $|j(t)|$ is Bernoulli distributed with a very small value of $p, \mathrm{SINR}_{\mathrm{LC}(3)} \approx \epsilon_{s}$ and $G_{\mathrm{LC}(3)} \approx 1+\alpha^{2} /\left(1-\alpha^{2}\right)$, proving the great interest of the L-C(3) MVDR beamformer in this case.

\section{Performance of $W L-C(q)$ and $L-C\left(q_{1}, q_{2}\right)$ MVDR beam- formers}

We consider in this Subsection, WL-C $\left(q_{1}\right)$ and L-C $\left(q_{1}, q_{2}\right)$ MVDR beamformers, $q_{1}, q_{2}=0, . ., 3$ and we analyze their performance in the presence of a single interference, i.e., from the total noise model (48). Using symbolic math toolboxes, it is possible to prove that $G_{\mathrm{WL}-\mathrm{C}\left(q_{1}\right)} / G_{\mathrm{WL}}$ and $G_{\mathrm{L}-\mathrm{C}\left(q_{1}, q_{2}\right)}$ follow the rational fraction form:

$$
G\left(\epsilon_{j}\right)=1+\frac{\alpha^{2} \beta^{6} \epsilon_{j}^{4}\left(a_{P-4} \epsilon_{j}^{P-4}+\ldots+a_{1} \epsilon_{j}+a_{0}\right)}{b_{P} \epsilon_{j}^{P}+\ldots+b_{1} \epsilon_{j}+b_{0}} .
$$

Here, $P=9$ and $P=11$ for $G_{\mathrm{WL}-\mathrm{C}\left(q_{1}\right)} / G_{\mathrm{WL}}$ and $G_{\mathrm{L}-\mathrm{C}\left(q_{1}, q_{2}\right)}$, respectively, whereas the coefficients $a_{i}$ and $b_{i}$ are very intricate expressions, functions of $\alpha, \beta$ and the total noise statistics appearing in $\mathbf{R}_{\tilde{n}}$. Again, $G_{\mathrm{L}-\mathrm{C}\left(q_{1}, q_{2}\right)}=$ $G_{\mathrm{WL}-\mathrm{C}\left(q_{1}\right)} / G_{\mathrm{WL}}=G_{\mathrm{WL}}=1$ if $\alpha=0, \beta=0$ or $\epsilon_{j}=0$. Moreover, we have verified that $G_{\mathrm{L}-\mathrm{C}\left(q_{1}, q_{2}\right)}=1$ and $G_{\mathrm{WL}-\mathrm{C}\left(q_{1}\right)} / G_{\mathrm{WL}}=1$ for a circular and an arbitrary Gaussian interference, respectively, which is consistent with the optimality of the L and WL MVDR beamformers respectively in such situations. Otherwise, we have proved in particular, from (74) and the results of Section IV-B, that for a strong non-filtered BPSK interference:

$$
\begin{aligned}
G_{\mathrm{WL}-\mathrm{C}(0)} & \approx G_{\mathrm{WL}-\mathrm{C}(1)} \approx G_{\mathrm{WL}-\mathrm{C}(3)} \approx G_{\mathrm{L}-\mathrm{C}(0,1)} \\
& \approx G_{\mathrm{L}-\mathrm{C}(0,2)} \approx G_{\mathrm{L}-\mathrm{C}(0,3)} \approx G_{\mathrm{L}-\mathrm{C}(1,3)} \\
& \approx G_{\mathrm{L}-\mathrm{C}(0)} \approx 1+\frac{\alpha^{2}}{1-\alpha^{2}}>G_{\mathrm{WL}} \approx G_{\mathrm{L}-\mathrm{C}(1)} \\
& \approx 1+\frac{\alpha^{2}}{2-\alpha^{2}}>G_{\mathrm{L}-\mathrm{C}(3)}>G_{\mathrm{L}-\mathrm{C}(2)} \approx 1,(75)
\end{aligned}
$$

whereas for a strong non-filtered QPSK interference:

$$
\begin{gathered}
G_{\mathrm{L}-\mathrm{C}(1,3)} \approx 1+\frac{\alpha^{2}}{1-\alpha^{2}}>G_{\mathrm{L}-\mathrm{C}(1)} \approx 1+\frac{\alpha^{2}}{2-\alpha^{2}} \\
>G_{\mathrm{L}-\mathrm{C}(3)}>G_{\mathrm{WL}}=G_{\mathrm{L}-\mathrm{C}(0)}=G_{\mathrm{L}-\mathrm{C}(2)}=1 .
\end{gathered}
$$

This result shows in particular that in this latter case, $\mathrm{SINR}_{\mathrm{L}-\mathrm{C}(1,3)} \approx \epsilon_{s}$ which proves the quasi-optimality (among the beamformers which use the total noise statistics only) of the L-C (1,3) MVDR beamformer for a strong nonfiltered QPSK interference. Finally, let us note that in all 
cases, the WL-C $(0,1,2,3)$ MVDR beamformer reaches at least the performance of the best WL-C $\left(q_{1}\right)$ and $\mathrm{L}-\mathrm{C}\left(q_{1}, q_{2}\right)$ MVDR beamformer and is thus quasi-optimal not only for strong non-filtered BPSK and QPSK interference but also for very impulsive interference, circular or not.

\section{Performance illustrations}

In order to illustrate the results of Sections IV-B and IV-C, we consider a two-element array with unit gain sensors and we assume that the SOI has a signal to noise ratio (SNR), $\pi_{s} / \eta_{2}$, equal to $10 \mathrm{~dB}$. This SOI is assumed to be corrupted by a single interference whose INR, $\pi_{j} / \eta_{2}$, is equal to $30 \mathrm{~dB}$. Under these assumptions, Fig. 2 displays, for a non-filtered BPSK interference, the variations of SINR $_{B}$ at the output of the B MVDR beamformers as a function of $\alpha$, for $\mathrm{B}=\mathrm{L}$, WL, $\mathrm{L}-\mathrm{C}(q), q=0, . .3, \mathrm{~L}-\mathrm{C}(1,3)$, WL$\mathrm{C}(0)$, WL-C $(0,1)$, WL-C $(0,1,3)$ and $\mathrm{WL}-\mathrm{C}(0,1,2,3)$. Note that this figure confirms the results (75), i.e., the equivalent performance of the $\mathrm{L}-\mathrm{C}(0), \mathrm{WL}-\mathrm{C}(0)$ and $\mathrm{L}-\mathrm{C}(1,3)$ MVDR beamformers and the better performance of the LC(0) MVDR beamformer with respect to the WL MVDR beamformer, itself better than the L-C(1), L-C(3) and L$\mathrm{C}(2)$ MVDR beamformers, the latter being equivalent to the Capon beamformer. Moreover, Fig. 2 shows the very weak information brought by the WL-C $(0,1)$, WL-C $(0,1,3)$ and WL-C $(0,1,2,3)$ MVDR beamformers with respect to the $\mathrm{L}-\mathrm{C}(0)$ or $\mathrm{L}-\mathrm{C}(1,3)$ MVDR beamformers which are quasioptimal.

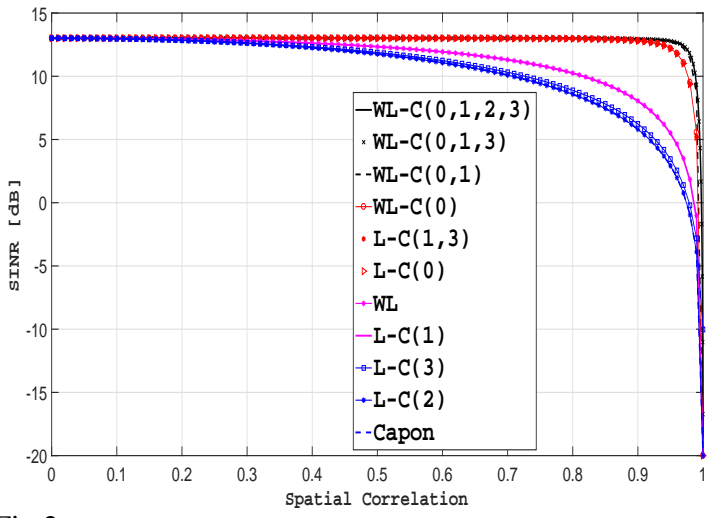

Fig.2 SINR $_{\mathrm{B}}$ as a function of $\alpha$, non-filtered BPSK interference.

Under the assumptions of Fig.2, Fig.3 displays the same variations as Fig.2, but for a non-filtered QPSK interference and for $\mathrm{B}=\mathrm{L}, \mathrm{L}-\mathrm{C}(q), q=1,3$ and L-C $(1,3)$. Again, this figure confirms the results (76), i.e., the better performance of the L-C $(1,3)$ MVDR beamformer with respect to the L-C $(1)$ MVDR beamformer, itself better than the L-C(3) MVDR beamformer, itself better than the Capon beamformer.

Under the assumptions of Fig.2, Fig.4 displays the variations of $G_{\mathrm{B}}$ at the output of the B MVDR beamformers as a function of $\alpha$, but for an impulsive circular interference, such that $|j(t)|$ follows a Bernoulli distribution with $p=0.001$. For this figure, $\mathrm{B}=\mathrm{L}-\mathrm{C}(1), \mathrm{L}-\mathrm{C}(1,3)$ and $\mathrm{L}-\mathrm{C}(0,1,2,3)$. The value $p=0.001$ gives the high value $\kappa_{j, c}=1000(11)$ and thus this figure confirms that in this case $G_{\mathrm{LC}(1)} \approx 1+\alpha^{2} /\left(1-\alpha^{2}\right)$ is quasioptimal. As a consequence, the beamformers L-C $(1,3)$ and $\mathrm{L}-\mathrm{C}(0,1,2,3)$ bring no further gains with respect to $\mathrm{L}-\mathrm{C}(1)$.

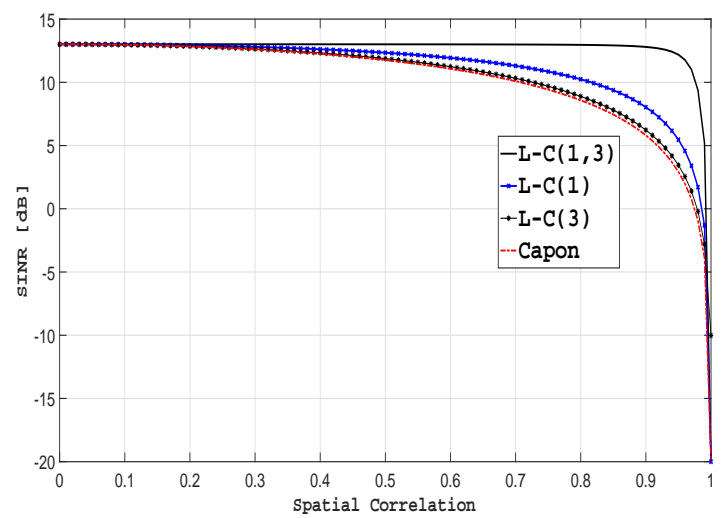

Fig.3 SINR $_{\mathrm{B}}$ as a function of $\alpha$, non-filtered QPSK interference.

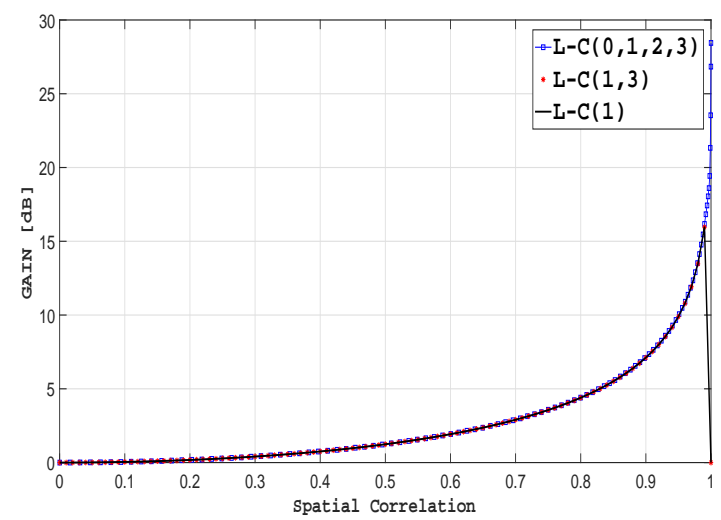

Fig. $4 G_{\mathrm{B}}$ as a function of $\alpha$, circular Bernoulli impulsive interference.

To show the interest of the proposed beamformers for moderate values of the INR, Fig. 5 shows, for $N=2$, a BPSK interference and several values of the modulus $\alpha$ of the spatial correlation coefficient between the SOI and interference, the variations, as a function of the input INR of the interference, of the SINR gain with respect to Capon beamformer, at the output of the WL-C $(0)$ beamformer. To complete these results, Fig. 6 shows similar variations but for a QPSK interference and at the output of the L$\mathrm{C}(1,3)$ beamformer. Note that these performance gains are independent of the SNR and are thus valid for arbitrary values of the SNR, weak or strong, as shown by expressions ((49), (51), (52) to (59), (74)). In other words, a given value of INR may be associated with a small, moderate or high value of the ratio INR/SNR. These figures show that the gain at the output of the proposed beamformers increases with the INR whatever the value of $\alpha$. However, this gain in performance may remain relatively high even for moderate INR. For example, for $\alpha=0.9$, Fig.5 and Fig.6 indicate that a SINR gain of 5, 4, 3, 2dB may be obtained for INR $=21,17,14,11 \mathrm{~dB}$ and $21.5,18.5,16.5$, $14 \mathrm{~dB}$, respectively for BPSK and QPSK interference, which correspond to classical values of INR for numerous appli- 
cations as explained hereafter. This shows that substantial gains in performance with respect to Capon beamformer may also be obtained for moderate values of the INR, hence the practical interest of the proposed beamformers even for moderate values of the INR. Note that for an array of $N=5$ antennas, similar gains would be obtained but for still lower values of the INR, as explained previously.

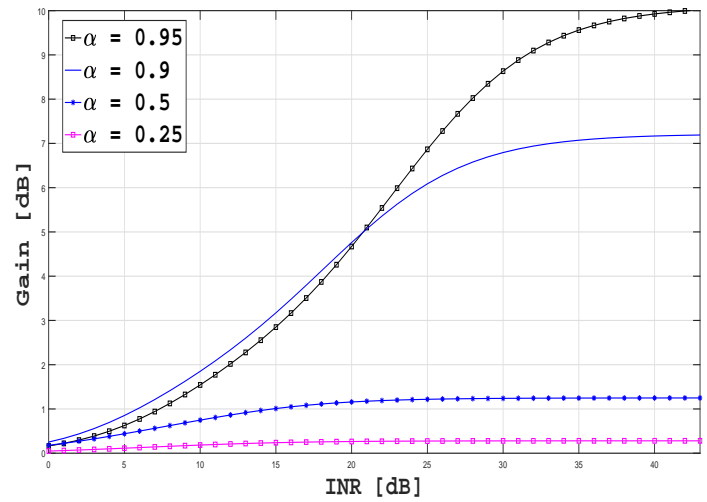

Fig. 5 Gain of the WL-C $(0)$ beamformer as a function of INR for a BPSK interference.

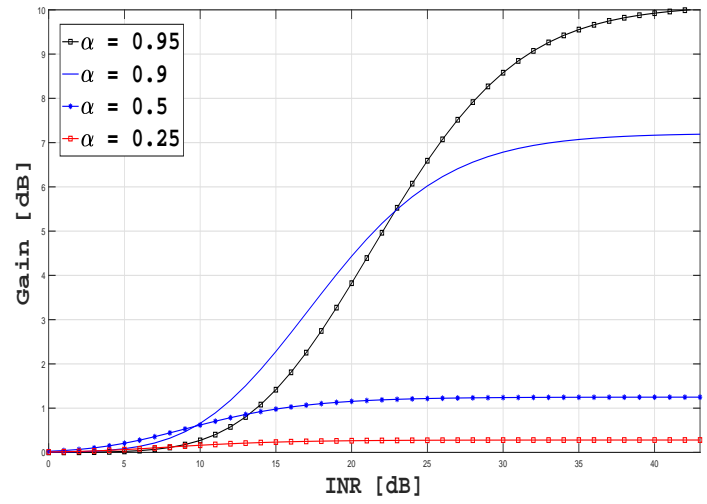

Fig.6 Gain of the L-C $(1,3)$ beamformer as a function of INR for a QPSK interference.

Note finally that in practice, both the INR and the nature of the interference may change, depending on the application. In particular, for military communications (HF, tactical, naval, airborne..), the interference may correspond to hostile jammers with arbitrary waveforms and power levels. In such situations, the INR may vary from a few $\mathrm{dB}$ to a few tens of $\mathrm{dB}$, depending on the power, the bandwidth and the distance of the jammer with respect to the SOI. For spectrum monitoring of HF and VUHF links, the probability to receive several sources, and/or several propagation paths of a given source, increases with the receiver bandwidth. Depending on parameters such as the transmitted power, the propagation channel, the bandwidth or the distance to the receiver of the received sources, the latter may be received over a very large power range, of a few tens of $\mathrm{dB}$. For such situations both the SNR and the INR may vary between a few $\mathrm{dB}$ to several tens of $\mathrm{dB}$. Strong dynamic ranges may also be encountered for metrology, or interference analysis, of the downlink of cellular networks, where the received signals correspond to signals emitted by different base station using the same frequency.

\section{ADAPTIVE IMPLEMENTATION}

As for the Capon beamformer, several adaptive implementations may be developed from the GSC structure of the third-order Volterra MVDR beamformers. We propose here to use an extension of the sample matrix inversion (SMI) algorithm [48] to implement (42). It consists to estimate the $\mathbf{R}_{\tilde{x}}$ matrix from the $K$ observation snapshots $\widetilde{\mathbf{x}}\left(k T_{e}\right)$, where $T_{e}$ is the sample period, by the empirical estimate given by

$$
\widehat{\mathbf{R}}_{\tilde{x}}=\frac{1}{K} \sum_{k=1}^{K} \widetilde{\mathbf{x}}\left(k T_{e}\right) \widetilde{\mathbf{x}}^{H}\left(k T_{e}\right)
$$

and then to compute an estimate, $\widehat{\mathrm{w}^{\prime}}{ }_{a, \text { opt }}$, of ${\widetilde{\mathbf{w}^{\prime}}}_{a, \text { opt }}$, given by:

$$
{\widehat{\mathbf{w}^{\prime}}}_{a, \text { opt }}=\left[\mathbf{K B}^{H} \widehat{\mathbf{R}}_{\tilde{x}} \mathbf{B} \mathbf{K}^{H}\right]^{-1} \mathbf{K B}^{H} \widehat{\mathbf{R}}_{\tilde{x}} \widetilde{\mathbf{w}}_{f} .
$$

For sufficiently oversampled and cycloergodic observations, $\widehat{\widehat{\mathbf{w}^{\prime}}}{ }_{a, \text { opt }}$ asymptotically converges towards $\widetilde{\mathbf{w}^{\prime}}{ }_{a, \text { opt }}$.

The theoretical analysis of the third-order Volterra SMI algorithm is beyond the scope of this paper and we simply illustrate its convergence through Monte-Carlo experiments. For this purpose, we consider again the total noise model (48) with $N=2$ and we assume that $\alpha=0.95$. The SOI and interference have the same waveform and are such that SNR $=10 \mathrm{~dB}, \mathrm{INR}=30 \mathrm{~dB}$ and $\phi_{s i}=\pi / 4$. The SINR at the output of a third-order Volterra beamformer implemented by the SMI algorithm from $K$ independent observation snapshots is defined by:

$$
\operatorname{SINR}(K)=\frac{\pi_{s}}{\left(\widetilde{\mathbf{w}}_{f}-\widehat{\mathbf{B w}}^{\prime}{ }_{a, \mathrm{opt}}\right)^{H} \mathbf{R}_{\tilde{x}}\left(\widetilde{\mathbf{w}}_{f}-{\widehat{\mathbf{B \mathbf { w } ^ { \prime }}}}_{a, \mathrm{opt}}\right)-\pi_{s}}
$$

Under these assumptions, Fig.7 shows, for a non-filtered QPSK SOI and a non-filtered BPSK interference, the variations, as a function of $K$, of the estimated mean value of $\operatorname{SINR}(K), \widehat{\mathrm{E}}(\operatorname{SINR}(K))$, computed over 1000 runs, at the output of several beamformers proposed in the paper or borrowed from the literature and corresponding to the Capon, MDDR [43], WL-MVDR [9], WL-MMSE [13], WL-MDDR [44], L-C $(0)$, L-C $(1,3)$ and WL-C $(0)$ beamformers. Fig.7 shows that for the considered scenario, the MDDR beamformer is not faster than the Capon beamformer, while the WL MDDR beamformer is slightly faster than the WL MVDR beamformer but not faster than the WL MMSE beamformer. Nevertheless, we verify that the steady state performance of the WL MDDR beamformer are upper-bounded by the performance of the theoretical WL MMSE beamformer, itself a bit higher than the performance of the theoretical WL MVDR beamformer for the considered scenario, itself better than the theoretical Capon beamformer since the interference is rectilinear. However, we note that the WL-C $(0), \mathrm{L}-\mathrm{C}(1,3)$ and $\mathrm{L}-\mathrm{C}(0)$ beamformers are much better than the WL MVDR beamformer for $K \geq 12$ and 
much better than the WL MDDR beamformer for $K \geq 8$, with a convergence speed decreasing as the number, $N_{i}$, of entries increases, hence the great interest of the proposed third-order beamformers with respect to the WL MDDR one in particular.

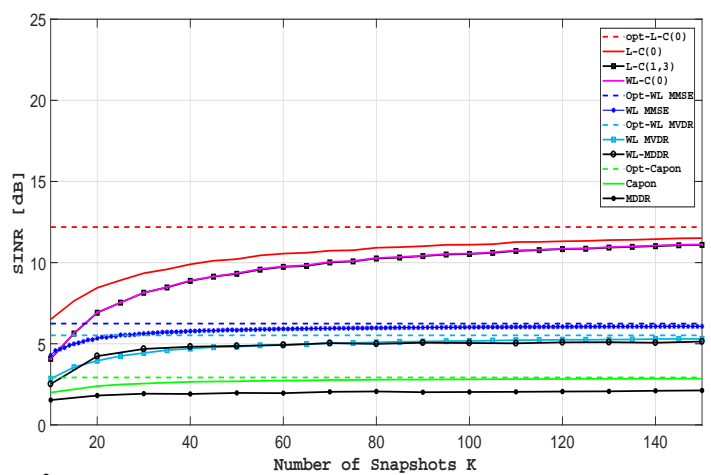

Fig.7 $\widehat{\mathrm{E}}(\operatorname{SINR}(K))$ as a function of $K$ for a non-filtered QPSK SOI and a non-filtered BPSK interference

\section{COMPlEXity ELEMENTS}

Due to a lack of place, we only give some complexity elements of the proposed third-order MVDR beamformers for a per-block strategy of adaptation, assuming the observations are stationary over the block. In such a case, the beamformers are estimated only one time per block of $K$ observation snapshots, which generates a complexity of $O\left(N^{9} / K\right)$ to generate one output sample. Moreover, for a given thirdorder MVDR beamformer, to ensure the invertibility of the correlation matrix estimate of the input vector, $\widetilde{\mathbf{z}}^{\prime}(t)$, of the adaptive part of its associated GSC structure, $K$ must be greater than or equal to the size, $N_{i}$, of $\widetilde{\mathbf{z}}^{\prime}(t)$. This means that $K$ must be necessarily at least equal to $O\left(N^{3}\right)$, which remains relatively low for small-scale systems $(1 \leq N \leq 5)$. This constraint generates, whatever the possible values of $K$, third-order beamformer complexities necessarily lower than $O\left(N^{6}\right)$, which remains very acceptable for small-scale systems. In practice, $K$ is often chosen as a multiple of $N_{i}$, i.e., $K=p N_{i}$, where $p$ is an integer such that $p \geq 1$.

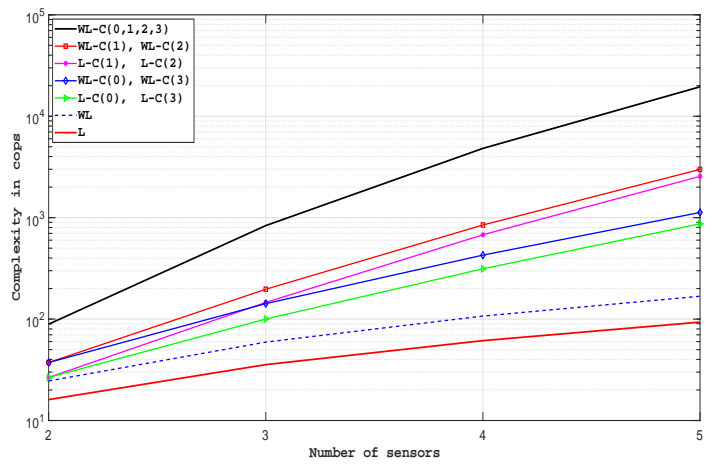

Fig. 8 Complexity of several first-order and third-order beamformers as a function of $N, p=20$

Under these assumptions, Fig.8 shows, for $p=20$, the variations, as a function of $N$, of the number of complex operations (cops) required to generate one output sample of several first and third-order beamformers. We note that for small-scale systems, the number of cops required by most of the third-order beamformers to generate one output sample does not exceed 1000, which remains very acceptable, hence the interest of the proposed beamformers also from a complexity point of view.

\section{CONCLUSION}

A family of third-order Volterra MVDR beamformers, containing L-cubic and WL-cubic MVDR beamformers, have been presented and analyzed in this paper, for the reception of an unknown SOI, whose waveform is unknown but whose steering vector in known, corrupted by potentially non-Gaussian and/or non-circular interference. These beamformers correspond to third-order extensions of the Capon or WL-MVDR 1 beamformers, allowing us to take into account the potential non-Gaussiannity and non-circularity of interference up to the SIO. These new beamformers are mainly developed for small-scale systems, having low spatial resolution, and for which the Capon beamformer may have limited performance in the presence of interference. Such systems are omnipresent for both civilian and military applications borrowed from radiocommunications, metrology or spectrum monitoring of the latter and for which the dynamic range of interference may reach a few tens of $\mathrm{dB}$. These beamformers do not require any a priori information about the interference and turn out to be particularly wellsuited for spectrum monitoring in circular or non-circular non-Gaussian contexts. Each of these beamformers has an equivalent GSC structure, allowing, for small-scale systems, its simple adaptive implementation with a very acceptable complexity from the extended block SMI algorithm. An analytical performance analysis of some L-cubic and WLcubic MVDR beamformers in the presence of one interference has been presented for $N=2$ antennas. It allows us to specify how these beamformers outperform the Capon and the WL MVDR 1 beamformers for circular and noncircular non-Gaussian interference respectively, depending on both the interference INR and the SO, FO and SIO interference statistics. This analysis enlightens the great interest of the proposed beamformers which outperform, in most situations, most of the beamformers of the literature such as the MDDR or WL MDDR beamformers. Further works about the proposed third-order MVDR beamformers may concern the behavior of the latter in the presence of multiple interference.

\section{APPENDIX}

\section{Proof of (52)}

For arbitrary steering vectors $\mathbf{s}=\left(1, e^{i \omega_{s}}\right)^{T}$ and $\mathbf{j}=$ $\left(1, e^{i \omega_{j}}\right)^{T}$ with $\omega_{s}, \omega_{j} \in[-\pi,+\pi]$, it is straightforward to prove that $\alpha=\left|\cos \left(\frac{\omega_{j}-\omega_{s}}{2}\right)\right|$ and the choice $\mathbf{w}_{f}=\frac{\mathbf{s}}{2}$ and 
$\left.\mathbf{u}_{1}=\frac{i e^{i \phi} \operatorname{sign}\left(\omega_{s}-\omega_{j}\right)}{\sqrt{2}}\left(e^{i\left(\omega_{j}-\omega_{s}\right) / 2},-e^{i\left(\omega_{j}+\omega_{s}\right) / 2}\right)\right)^{T}$ give the simplified model:

$$
\begin{aligned}
y_{f}(t) & \stackrel{\text { def }}{=} \quad \mathbf{w}_{f}^{H} \mathbf{x}(t)=s(t)+\frac{1}{\sqrt{2}}\left(\alpha j^{\prime}(t)+n_{1}(t)\right) \\
z(t) & \stackrel{\text { def }}{=} \quad \mathbf{u}_{1}^{H} \mathbf{x}(t)=\beta j^{\prime}(t)+n_{2}(t)
\end{aligned}
$$

with $\beta=\stackrel{\text { def }}{=} \sqrt{1-\alpha^{2}}$ and $j^{\prime}(t) \stackrel{\text { def }}{=} \sqrt{2} j(t) e^{-i \phi}$ (where $\left.\frac{\mathbf{s}^{H} \mathbf{j}}{2}=\alpha e^{i \phi}\right)$ and where $n_{1}(t)$ and $n_{2}(t)$ are independent zero-mean circular Gaussian distributed with variance $\eta_{2}$.

By the orthogonal projection theorem, $<\mathrm{E}\left|y_{f}^{2}(t)\right|>=<$ $\mathrm{E}\left|\widehat{y}_{f}^{2}(t)\right|>+<\mathrm{E}\left|y^{2}(t)\right|>$ and thus the SINR at the output of the GSC structure is given by:

$$
\begin{aligned}
\operatorname{SINR}_{\mathrm{L}-\mathrm{C}(q)} & =\frac{<\mathrm{E}\left|s^{2}(t)\right|>}{<\mathrm{E}\left|y^{2}(t)\right|>-<\mathrm{E}\left|s^{2}(t)\right|>} \\
& =\frac{<\mathrm{E}\left|s^{2}(t)\right|>}{<\mathrm{E}\left|y_{f}^{2}(t)\right|>-<\mathrm{E}\left|\widehat{y}_{f}^{2}(t)\right|>-<\mathrm{E}\left|s^{2}(t)\right|>} \\
& =\frac{\pi_{s}}{\alpha^{2} \pi_{j}+\frac{\eta_{2}}{2}-<\mathrm{E}\left|\widehat{y}_{f}^{2}(t)\right|>} .
\end{aligned}
$$

To compute $<\mathrm{E}\left|\widehat{y}_{f}^{2}(t)\right|>$, we use a Gram-Schmidt orthonormalization of the couple $\left(z(t), z_{3, q}(t)\right)$, which gives the unit variance uncorrelated random variables:

and

$$
v_{1}(t)=\frac{z(t)}{<\mathrm{E}\left|z^{2}(t)\right|>}
$$

$$
v_{2}(t)=\frac{z_{3, q}(t)-\left(\frac{<\mathrm{E}\left(z^{*}(t) z_{3, q}(t)\right)>}{<\mathrm{E}\left|z^{2}(t)\right|>}\right) z(t)}{\sqrt{<\mathrm{E}\left(\left|z_{3, q}(t)-\left(\frac{<\mathrm{E}\left(z^{*}(t) z_{3, q}(t)\right)>}{<\mathrm{E}\left|z^{2}(t)\right|>}\right) z(t)\right|^{2}>\right)}} .
$$

In this new basis, the output of the optimal linear filtering of $\widetilde{\mathbf{z}}(t)=\left(z(t), z_{3, q}(t)\right)^{T}$ is given by:

$$
\widehat{y}_{f}(t)=<\mathrm{E}\left[v_{1}^{*}(t) y_{f}(t)\right]>v_{1}(t)+<\mathrm{E}\left[v_{2}^{*}(t) y_{f}(t)\right]>v_{2}(t),
$$

which gives $<\mathrm{E}\left|\widehat{y}_{f}^{2}(t)\right|>=\left|<\mathrm{E}\left[v_{1}^{*}(t) y_{f}(t)\right]>\right|^{2}+\mid<$ $\mathrm{E}\left[v_{2}^{*}(t) y_{f}(t)\right]>\left.\right|^{2}$. Noting that for the Capon beamformer $\widehat{y}_{f}(t)=<\mathrm{E}\left[v_{1}^{*}(t) y_{f}(t)\right]>v_{1}(t)$ implying $<\mathrm{E}\left|\widehat{y}_{f}^{2}(t)\right|>=\mid<$ $\mathrm{E}\left[v_{1}^{*}(t) y_{f}(t)\right]>\left.\right|^{2}$, the SINR at its output is given by

$$
\mathrm{SINR}_{\mathrm{L}}=\frac{\pi_{s}}{\alpha^{2} \pi_{j}+\frac{\eta_{2}}{2}-\left|<\mathrm{E}\left[v_{1}^{*}(t) y_{f}(t)\right]>\right|^{2}} .
$$

Consequently, comparing the expressions of $\operatorname{SINR}_{\mathrm{L}-\mathrm{C}(q)}$ and $\mathrm{SINR}_{\mathrm{L}}$ given in (82) and (85), we deduce that the gain $G_{\mathrm{L}-\mathrm{C}(q)}$ in SINR with respect to the Capon beamformer satisfies the relation:

$$
\mathrm{G}_{\mathrm{L}-\mathrm{C}(q)}^{-1}=1-\frac{\operatorname{SINR}_{\mathrm{L}}}{\pi_{s}}\left|<\mathrm{E}\left[v_{2}^{*}(t) y_{f}(t)\right]>\right|^{2} \leq 1 .
$$

Replacing respectively, $v_{2}(t)$ by (83) where $z(t)$ is given by (81), and $y_{f}(t)$ by (80), the values of $\left|<\mathrm{E}\left[v_{2}^{*}(t) y_{f}(t)\right]>\right|^{2}$ are deduced for $q=0,1,2,3$ after cumbersome algebra derivations and thus (59) is derived from (86). Then using (49), the general expression (52) is proved.

\section{REFERENCES}

[1] B.D. Van Veen, K.M. Buckley, "Beamforming: A versatile approach to spatial filtering," IEEE ASSP Magazine, pp. 4-24, April 1988.

[2] J. Capon, R.J. Greenfield, and R.J. Kolker, "Multidimensional maximum likelihood processing of a large aperture seismic array," Proc. IEEE, vol. 55, no. 2, pp. 191-211, Feb. 1967.

[3] O.L. Frost, III, "An algorithm for linearly constrained adaptive array processing," Proc. IEEE, vol. 60, no. 8, pp. 926-935, Aug. 1972.

[4] L.J. Griffiths, C.W. Jim, "An alternative approach to linearly constrained adaptive beamforming," IEEE Trans. Ant. Prop., vol AP-30, no.1, pp. 27-34, Jan. 1982.

[5] J. Li and P. Stoica, Robust adaptive beamforming, John Wiley \& Sons, Inc., Hoboken, New Jersey, 2006.

[6] B. Picinbono, P. Chevalier, "Widely linear estimation with complex data," IEEE Trans. Signal Process., vol. 43, pp. 2030-2033, Aug. 1995.

[7] B. Picinbono, "On circularity," IEEE Trans. Signal Process., vol. 42, no. 12, pp. 3473-3482, Dec 1994.

[8] P.O. Amblard, M. Gaeta, and J.L. Lacume, "Statistics for complex variables and signals - Part I and II," Signal Processing, Elsevier, vol. 53, no. 1, pp. 1-25, Aug. 1996.

[9] P. Chevalier, A. Blin, "Widely linear MVDR beamformer for the reception of an unknown signal corrupted by noncircular interferences," IEEE Trans. Signal Process., vol. 55, pp. 5323-5336, Nov. 2007.

[10] T. Mc Whorter, P. Schreier, "Widely linear beamforming," Proc. 37th Asilomar Conference, Pacific Groeve (USA), pp. 753-759, Nov. 2003.

[11] P. Chevalier, J.P. Delmas, and A. Oukaci, "Optimal widely linear MVDR beamforming for noncircular Signals," Proc. ICASSP, Taipei (Taiwan), April 2009

[12] P. Chevalier, J.P. Delmas, and A. Oukaci, "Properties, performance and practical interest of the Widely Linear MMSE beamformer for nonrectilinear signals," Signal Processing, Elsevier, vol. 97, pp. 269281, April 2014.

[13] P. Chevalier and F. Pipon, "New insights into optimal widely linear array receivers for the demodulation of BPSK, MSK and GMSK signals corrupted by non circular interferences - Application to SAIC," IEEE Trans. Signal Process., vol 54, no. 3, pp. 870-883, March 2006.

[14] D. Xu, L. Huang, X. Xu, and Z. Ye, "Widely linear MVDR beamformers for noncircular signals based on time-averaged secondorder noncircularity coefficient estimation", IEEE Trans. Vehicular Technology, vol. 62, no. 7, pp. 3219-3227, Sept. 2013.

[15] G. Wang, J.P. Lie, and C.S. See, "A robust approach to optimum widely linear MVDR beamformer", Proc. ICASSP, Kyoto (Japan), March 2012.

[16] F. Wen, Q. Wan, H. Wei, R. Fan, and Y. Luo, "Robust Capon beamforming exploiting the second-order noncircularity of signals", Signal Process., Elsevier, vol. 102, pp. 100-111, 2014.

[17] D. Xu, C. Gong, S. Cao, X. Xu, and Z. Ye, "Robust widely linear beamforming based on spatial spectrum of noncircularity coefficient", Signal Process., Elsevier, vol. 104, pp. 167-173, Nov. 2014.

[18] J. Zhang, L. Huang, L. Zhang, B. Zhang, and Z. Ye, "Robust widely linear beamformer based on a projection constraint", Proc. ICASSP, Brisbane (Australia), pp. 2509-2513, April 2015.

[19] A. Hakkarainen, J. Werner, K.R. Dandekar, and M. Valkama, ”Widely linear beamforming and RF impairment suppression in massive antenna arrays", Journal of Communications and Networks, vol. 15, no. 4, pp. 383-397, Aug. 2013.

[20] N. Song, W.U. Alokozai, R.C. De Lamare, and M. Haardt, "Adaptive widely linear reduced-rank beamforming Based on joint iterative optimization", IEEE Signal Process. Letters, vol. 21, no. 3, pp. 265269, March 2014

[21] J. Benesty, J. Chen, and Y. Huang, "Binaural noise reduction in the time domain with a stereo setup", IEEE Trans. Audio Speech Lang. Process., vol. 19, no. 8, pp. 2260-2272, Nov. 2011.

[22] J. Chen and J. Benesty, "On the time-domain widely linear LCMV filter for noise reduction with a stereo system", IEEE Trans. Audio Speech Lang. Process., vol. 21, no. 7, pp. 1343-1354, July 2013.

[23] M.S. Arulampalam, S. Maskell, N. Gordon, and T. Clapp, "A tutorial on particle filters for online nonlinear/non-Gaussian Bayesian tracking”, IEEE Trans. Signal Process., vol. 50, no. 2, pp. 174-188, Feb. 2002. 
[24] P.M. Djuric, J.H. Kotecha, J. Zhang, Y. Huang, T. Ghirmai, M.F. Bugallo, and J. Miguez, "Particle Filtering", IEEE Signal Process. Mag., vol. 20, no. 5, pp. 19-38, 2003.

[25] L.M. Garth and H.V. Poor, "Narrowband interference suppression in impulvive channels", IEEE Trans. Aerosp. Electron. Syst., vol; 28, no. 1, pp. 15-34, 1992.

[26] A. Mohammadi and K.N. Plataniotis, "Complex-valued Gaussian sum filter for nonlinear filtering of non-Gaussian/non-circular noise", IEEE Signal Proc. Letters, vol. 22, no. 4, pp. 440-444, April 2015.

[27] C.Q. Xu, C.L. Law, and S. Yoshida, "Interference rejection in nonGaussian noise for satellite communications using non-linear beamforming", Int. Journal on Satellite Commun. Networking, vol. 21, pp. 13-22, 2003.

[28] P. Chevalier, P. Duvaut, B. Picinbono, "Le filtrage de Volterra transverse réel et complexe en traitement du signal," Traitement du Signal, "Non linéaire et non gaussien," vol. 7, n. 5, pp 451-476, 1990.

[29] P. Chevalier, P. Duvaut, and B. Picinbono, "Complex transversal Volterra filters optimal for detection and estimation", Proc ICASSP, Toronto (Canada), pp. 3537-3540, 1991.

[30] M. Schetzen, The Volterra and Wiener theory of non linear systems, Wiley, New-York, 1980.

[31] B. Pincibono and P. Duvaut, "Optimal linear quadratic systems for detection and estimation," IEEE Trans. Info. Theory, vol. 34, pp. 304311, March 1988.

[32] T. Koh and E.J. Powers, "Second order Volterra filtering and its application to non linear system identification," IEEE Trans. Acou. Speech Signal Proc., vol. 33, no. 6, pp. 1445-1455, Dec. 1985.

[33] O. Agazzi, D.G. Messerschmitt, and D.A. Hodges, "Non linear echo cancellation of data signals," IEEE Trans. Commun., vol. 30, no. 11, pp. 2421-2433, Nov. 1982.

[34] R.D. Nowak and B.D. Van Veen, "Volterra filter equalization : a fixed point approach," IEEE Trans. Signal Process., vol. 45, no. 2, pp. 377388, Feb. 1997.

[35] S. Benedetto, E. Bigieri, and R. Daffara, "Modeling and performance evaluation of non-linear satellite links - A Volterra series approach," IEEE Trans. Aerosp. Elect. Syst., vol. 15, pp. 494-507, July 1979.

[36] C. Crespo-Cadenas, M.J. Madero-Ayora, J. Reina-Tosina, J.A. Becerra-Gonzales, "Formal deduction of a Volterra series model for complex-valued systems," Signal Processing, vol. 131, 2017.

[37] G. Mileounis and N. Kalouptsidis, "Blind identification of secondorder Volterra systems with complex random inputs using higher order cumulants," IEEE Trans. Signal Process., vol. 57, no. 10, pp. 41294135, Oct. 2009

[38] P. Chevalier and B. Picinbono, "Complex linear-quadratic systems for detection and array processing," IEEE Trans. Signal Process., vol. 44, no. 10 , pp. 2631-2634, Oct. 1996.

[39] A. Souloumiac, P. Chevalier, and C. Demeure, "Improvement in nonGaussian jammers rejection with a non linear spatial filter," Proc ICASSP, Minneapolis (USA), pp. 670-673, April 1993.

[40] P. Comon, R. Liu, and D. Slock, "Path-wise wide-sense polynomial receiver for UMTS communications," Proc. 39th Allerton Conf. Com. Contr. Comput., Allerton, Illinois (USA), Oct. 2001.

[41] M. Sadok, JP. Delmas, P. Chevalier, "Enhanced Single-Antenna Interference Cancellation from MMSE third-order complex Volterra filters," Proc. ICASSP, New-Orleans (USA), March 2017.

[42] L. Antilla and M. Valkama, "Blind signal estimation in widely-linear signal models with fourth-order circularity: Algorithms and application to receiver I/Q calibration," IEEE Signal Process. Letters, vol. 20, no. 3, pp. 221-224, March 2013

[43] X. Jiang, W.J. Zeng, A. Yasotharan, H.C. So, and T. Kirubarajan, "Minimum dispersion beamforming for non-Gaussian signals," IEEE Trans. Signal Process., vol. 62, no. 7, pp. 1879-1893, April 2014.

[44] L. Huang, J. Zhang, L. Zhang, and Z. Ye, "Widely linear minimum dispersion beamforming for sub-Gaussian non-circular signals", Signal Processing, vol. 122, n. 7, pp. 123-128, 2016.

[45] P. Chevalier, A. Oukaci, and J.P. Delmas, "Third-order widely nonlinear Volterra MVDR beamforming," Proc. ICASSP, Prague (Czech Republic), May 2011.

[46] P. Chevalier, L. Albera, A. Ferreol, and P. Comon, "On the virtual array concept for higher order array processing," IEEE Trans. Signal Process., vol. 53, no. 4, pp. 1254-1271, April 2005.
[47] D.A. Harville, Matrix algebra from a statistician's perspective, Springer-Verlag, New York, 1997.

[48] I.S. Reed, J.D. Mallet, and L.E. Brennan, "Rapid convergence rate in adaptive arrays," IEEE Trans. Aerosp. Electron. Syst., vol. 10, no. 6 , pp. 853-863, Nov. 1974.

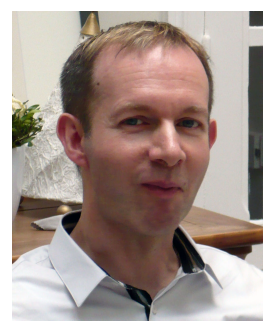

Pascal Chevalier (M'18) received the M.Sc. degree from Ecole Nationale Supérieure des Techniques Avancées, Paris, France, in 1985, the DEA degree in automatic and signal processing from Jussieu university (Paris VII), Paris, France, in 1987, the Ph.D. degree from South-Paris University (Paris XI), Paris, France, in 1991 and the Habilitation à Diriger des Recherches degree from Marne-La-Vallée University, Champs-sur-Marne, France, in 2009. Since 1991, he has been with Thales Communications-Security (France), where he has shared industrial activities (studies, experimentations, expertise, management), teaching activities both in French engineer schools (ESE, ENST, ENSTA, ESIEE) and French Universities (Cergy-Pontoise) and research activities. Since 2000, he has also been acting as a Technical Manager and an Architect of the array processing subsystem as part of a national program of military satellite telecommunications. He has been a Thales Expert since 2003. Since 2010, he has also been with the Conservatoire National des Arts et Métiers, Paris, France, as a Professor holder of the Electronic Chair. His current research interests include signal processing and array processing techniques for spectrum monitoring and digital communications, including second and higher order direction finding and beamforming, interference mitigation, widely linear and FRESH processing, equalization, nonorthogonal multicarrier waveforms, and MIMO systems. He is author or co-author of 29 patents and about 150 publications (Journal, Conferences and Chapters of books). He has been a member of the THOMSON-CSF Technical and Scientifical Council between 1995 and 1998. He co-received the 2003 "Science and Defense" Award from the french Ministry of Defence for its work as a whole about array processing for military radiocommunications. Since 2003, he has been an Associate Editor for EURASIP Journal of Wireless Communications and Networking and Co-technical Chairman of ISWCS'12 Symposium. He is currently a member of the EURASIP Special Area Team related to Signal Processing for Multisensor Systems, a member of the IEEE Sensor Array and Multichannel Technical Committee and an emeritus member of the Societé des Electriciens et des Electroniciens.

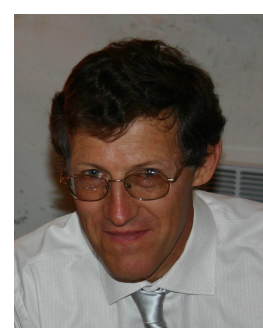

Jean Pierre Delmas (M'00-SM'06) was born in France in 1950. He received the engineering degree from Ecole Centrale de Lyon, Lyon, France in 1973, the Certificat d'Etudes Supérieures from Ecole Nationale Supérieure des Télécommunications, Paris, France in 1982 and the Habilitation à diriger des recherches degree from the University of Paris XI, Orsay, France in 2001. Since 1980, he has been with Telecom SudParis, where he is currently a Professor with the CITI Department. He was the Deputy Director (2005-2010) and the Director (2011-2014) of UMR 5157 (CNRS laboratory). His teaching and research interests include statistical signal processing with application to communications and antenna array. He was an Associate Editor for the IEEE Transaction on Signal Processing (20022006) and (2010-2014), and currently for Signal Processing (Elsevier). From 2011 to 2016, he was a member of the IEEE Sensor Array and Multichannel Technical Committee.

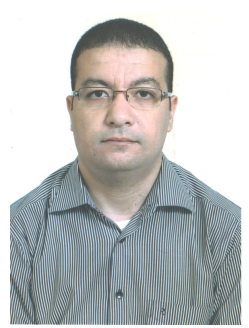

Mustapha Sadok received the $\mathrm{PhD}$ degree in applied mathematics and signal processing from Telecom SudParis, France, in December 2017. He is currently an Assistant Professor at Institut National des Télécommunications et des TIC d'Oran (INTTIC) Algeria. His research interests are in signal processing for communications and radio monitoring. 